\title{
Initiation of a Runaway Greenhouse in a Cloudy Column
}

\author{
MAX POPP \\ Max Planck Institute for Meteorology, and International Max Planck Research School on Earth System Modelling, \\ Hamburg, Germany
}

HAUKE SCHMIDT AND JOCHEM MAROTZKE

Max Planck Institute for Meteorology, Hamburg, Germany

(Manuscript received 8 February 2013, in final form 5 September 2014)

\begin{abstract}
A one-dimensional radiative-convective equilibrium model is used to investigate the influence of clouds on the onset of a runaway greenhouse under strong solar forcing. By comparing experiments with clear-sky conditions (clouds are transparent to radiation) to experiments with full-sky conditions (clouds are radiatively active), the authors find that the critical solar irradiance that is necessary to trigger a runaway greenhouse is increased from around 1.15-1.20 times the present-day total solar irradiance (TSI) on Earth $S_{0}$ for clear-sky conditions to around $1.40-1.45 S_{0}$ for full-sky conditions. Cloud thickness increases with TSI, leading to a substantially higher albedo, which in turn allows the climate to remain in equilibrium for markedly higher values of TSI. The results suggest that steady states with sea surface temperatures higher than $335 \mathrm{~K}$ exist for a large range of TSI. The thickening clouds in these states do not reduce the outgoing longwave radiation any more, implying that the thickening of clouds increases only their shortwave effect. This mechanism allows the column to remain in balance even at high sea surface temperatures. The authors find double equilibria for both clear-sky and full-sky conditions, but the range for which they occur extends to considerably higher values of TSIs for full-sky conditions. Moreover, when clouds are included in the radiative transfer calculations, climate instabilities are no longer caused by longwave effects but by the cloud albedo effect.
\end{abstract}

\section{Introduction}

In the 1960s, the idea was advanced that, under strong radiative forcing, a runaway water vapor feedback may occur, which may lead to the evaporation of all oceans on an Earthlike planet (e.g., Gold 1964; Komabyashi 1967; Ingersoll 1969) - the runaway greenhouse. The idea was used to explain how Venus could have lost most of its water and could have ended up with the inhospitable atmosphere that it has today. Even though 50 years have passed, the role of clouds in the context of the runaway greenhouse has, until recently, received little attention. Clouds cool the climate on present-day Earth and recent results suggest that, without clouds, Earth might well be on the verge of a runaway greenhouse (Goldblatt et al. 2013). Hence, this paper explores the role of clouds in the initiation of a runaway greenhouse.

Corresponding author address: Max Popp, Max Planck Institute for Meteorology, Bundesstraße 53, 20146 Hamburg, Germany.

E-mail: max.popp@mpimet.mpg.de
Vardavas and Carver (1985) used a simple cloud model of one layer to study the evolution of cloud properties with increasing surface temperatures. They assumed that the cloud cover and the cloud optical depth were proportional to the atmospheric water vapor content. Other studies made use of models with a constant cloud layer as well or investigated the influence of clouds tentatively for single cloud layers at different temperature and pressure levels (e.g., Kasting 1988). The radiativeconvective model used by Rennó et al. (1994) and Rennó (1997) included the thermodynamic properties of convective clouds, but either the radiative properties of the clouds were neglected or a fixed cloud cover was assumed.

More recently, Boer et al. (2005) studied the influence of strong solar forcings on Earth's climate with the National Center for Atmospheric Research (NCAR) Climate Systems Model (CSM), version 1.4, general circulation model (GCM). The cloud cover decreased with increasing temperature, causing the temperature to run away. This result stands in contrast to the expectation by Vardavas and Carver (1985) that cloud cover increases with increasing 
temperatures. The GCM simulation failed, however, before the average surface temperature exceeded $340 \mathrm{~K}$; therefore, only a relatively small range of temperatures could be explored. Heinemann (2009) found as well that the cloud cover decreased with increasing surface temperature in the ECHAM5 Max Planck Institute Ocean Model (MPI-OM) GCM. The surface temperatures also ran away for high temperatures, but only because, as a result of the prescribed concentrations of ozone, high concentrations of ozone came to lie in the troposphere as the tropopause climbed. This runaway behavior was, however, likely a direct consequence of the limitations of the radiation code in the applied GCM. Abe et al. (2011) studied the habitable zone of dry Earthlike planets with no oceans but including soil moisture. They used a GCM and included the radiative properties of clouds, but they did not focus on the influence of the clouds on the onset of a runaway greenhouse. As in Boer et al. (2005) and Heinemann (2009), the cloud cover decreased with increasing temperatures in Abe et al. (2011). Wolf and Toon (2014) and Leconte et al. (2013) have very recently investigated Earth under strong solar forcing with two different GCMs. Wolf and Toon (2014) only considered a range of surface temperatures up to $320 \mathrm{~K}$ and did not find any indication of a runaway greenhouse up to that temperature. Leconte et al. (2013) found that, as the steady-state global-mean surface temperature exceeds $290 \mathrm{~K}$, the net cloud forcing decreases with increasing temperature and then remains constant with increasing steady-state global-mean surface temperature as $320 \mathrm{~K}$ is exceeded. Since a steady state can be attained in their model for global-mean surface temperatures as high as $340 \mathrm{~K}$, clouds appear not to have any direct effect on the onset of a runaway greenhouse. However, the evolution of cloud properties is not presented for the transient simulations in which a runaway greenhouse occurs. Hence, it is still unknown whether clouds may play a role in maintaining the radiative imbalance at even higher surface temperatures.

Here we investigate the evolution of clouds and especially of the radiative properties of clouds on the onset of a runaway greenhouse. We use a one-dimensional radiative-convective equilibrium model with a complex hydrological cycle, including a scheme for the representation of cloud cover. Our model differs from all previous one-dimensional studies in that it computes self-consistently the location of clouds, their fractional cover, their microphysical properties, and their radiative properties. This allows us to explore the behavior of the column under strong solar forcings for clear-sky conditions (CSC; clouds are transparent to radiation) and for full-sky conditions (FSC; with full cloud-radiation interaction), for sea surface temperatures (SST) of up to $120^{\circ} \mathrm{C}(393.15 \mathrm{~K})$. Our approach permits us to quantify the effect of clouds and to estimate their influence on the value of the total solar irradiance (TSI) that is necessary to trigger a runaway greenhouse. Moreover, we can explore the possibility of equilibria at high temperatures, as found by Rennó (1997). Ensembles of simulations give us the possibility to assess the robustness of our results.

This paper is organized as follows. We start with a discussion of the energy balance in the context of the runaway greenhouse in section 2 before describing the single-column model, including its limitations, and the experimental setup in section 3 . The results are presented and described in section 4 . We discuss the behavior of the clouds and the emergence of multiple equilibria in detail in section 5. To assess the robustness of our main results, we present and discuss the results of three sets of sensitivity experiments in section 6 . We compare our results to previous studies in section 7 , and we finally state our conclusions in section 8 .

\section{Energy balance and the runaway greenhouse}

We start with some considerations of the energy balance at the top of the atmosphere. The crucial mechanism for a runaway greenhouse to occur is that the outgoing longwave radiation (OLR) decouples from the SST as the atmosphere becomes optically thick in the infrared (Nakajima et al. 1992; Goldblatt and Watson 2012). As a consequence, the OLR does not change anymore with SST and is hence bounded. If the absorbed solar radiation exceeds the upper bound of OLR, an uncompensated gain of energy occurs-the runaway greenhouse.

The temporal average of the steady-state energy balance at the top of the atmosphere can be written as

$$
\left(1-\alpha_{e}\right) \bar{S}=\overline{I_{0}},
$$

where the overbar denotes the temporal averages, $I_{0}$ is the OLR, $S$ is the TSI, and $\alpha_{e}$ is the effective albedo, defined as

$$
\alpha_{e}=\frac{\bar{S}_{R}}{\bar{S}},
$$

with $S_{R}$ the reflected shortwave radiation at the top of the atmosphere. The advantage of using the effective albedo is that, unlike the albedo, the effective albedo is a direct measure for the absorbed energy. For example, the value of the albedo during night has no influence on the energy budget.

We now discuss different assumptions on the optical properties of the atmosphere. We start by assuming that 
the atmosphere absorbs longwave radiation but no shortwave radiation, and that clouds are transparent to all radiation. Without atmospheric shortwave absorption, the effective albedo, and hence $\bar{S}_{R}$, remain constant. According to Eq. (1), if the TSI is increased, an equilibrium can only exist if the OLR increases accordingly. It follows in this case that if the OLR is bounded and if $\left(1-\alpha_{e}\right) \bar{S}$ exceeds the maximum that the OLR can attain, then a runaway greenhouse occurs.

We assume next that the atmosphere also absorbs shortwave radiation but that the clouds are still transparent to radiation. This is our CSC setup (see section $3 b$ ). The reflected shortwave radiation can now change with temperature, because the amount of atmospheric water vapor changes and because water vapor absorbs solar radiation. Hence, the reflected shortwave radiation will decrease with increasing SST (at least in the range of temperatures that we consider). If the TSI is increased, then an equilibrium can only be attained if the OLR compensates not only for the increase in TSI, but also for the reduction of reflected shortwave radiation. Therefore, the atmosphere is more susceptible to a runaway greenhouse in CSC than in the case without solar absorption by the atmosphere.

Finally, we assume that clouds interact with radiation as in our FSC setup. Clouds generally reduce the OLR, depending on the height at which the cloud top is located. Since the surface in our model is assumed to consist of liquid water, clouds always lead to an increase in albedo. For present-day Earth, high clouds tend to warm the climate, because they lower the OLR considerably, while being too thin for their albedo to be large. Low clouds tend to cool the climate because they have a small influence on the OLR, whereas their albedo is quite high. So, depending on whether the cloudiness increases or decreases, the level at which clouds form, and whether the shortwave or the longwave effect dominates, clouds may have a stabilizing or a destabilizing effect.

\section{Experimental design}

\section{a. Model}

We employ a modified single-column version of the general circulation model ECHAM6 (Stevens et al. 2013), which we couple to a mixed-layer ocean. We do not discuss the entire model here but give a short general introduction as well as a summary of the radiation and the convection schemes, the hydrological cycle, the cloudmicrophysical scheme, and the cloud-cover scheme. The column consists of 95 vertical layers and is energetically isolated. Hence, energy can only leave and enter the column in the form of radiation at the top of the atmosphere.
Several physical formulations of thermodynamic variables implemented in ECHAM6 are based on the assumption that the mass of water vapor in the atmosphere is negligible compared to the mass of dry air. In this case, the pressure of a column of dry air $p$ (which is assumed to be equal to the total pressure in ECHAM6) corresponds closely to the pressure of a column of moist air $P$, and the volume mixing ratio of water vapor corresponds closely to the specific humidity. However, if water vapor becomes a major constituent of the atmosphere, these approximations may lead to considerable errors. Therefore, we use more generalized formulations to allow for a more precise representation of thermodynamic variables. We include the mass of water vapor when calculating the total pressure, and we omit all approximations where small specific humidities were assumed (as, for example, in the calculation of density). Furthermore, we fix two inconsistencies in the original version of the model that caused energy not to be conserved.

If the tropopause climbs, then regions with high ozone concentrations may come to lie in the troposphere in ECHAM6, because ozone concentrations are prescribed to climatological values. High ozone concentrations could, however, not occur in the presence of tropospheric water vapor concentrations. Therefore, we limit the tropospheric ozone concentrations to a volume mixing ratio of $1.5 \times 10^{-7}$. As a consequence, ozone is taken out of the atmosphere if the tropopause rises to levels where the climatological values would exceed this limit.

\section{1) RADIATION}

The employed radiation scheme is the Rapid Radiative Transfer Model (RRTMG) (Mlawer et al. 1997; Iacono et al. 2008). It uses the correlated- $k$ method to solve the radiative transfer equations in the two-stream approximation. The shortwave radiation spectrum is divided into 14 bands, and the longwave radiation spectrum is divided into 16 bands. Since the lookup tables of the molecular absorption coefficients are designed for a limited range of temperatures only, we perform an exponential extrapolation for temperatures up to $400 \mathrm{~K}$ for the longwave radiation scheme. We apply the same extrapolation scheme to the lookup tables for the absorption coefficients of the water vapor self-broadened continuum in the shortwave radiation scheme, but we keep the original linear extrapolation scheme for all the other absorption coefficients. However, the exponential extrapolation likely becomes increasingly inaccurate the further the temperature gets from the range of the original lookup tables of the absorption coefficients.

We introduce the water vapor self-broadened continuum in the upper atmosphere radiation calculations, since water vapor concentrations increase above $100 \mathrm{hPa}$ 
as the temperatures rise. Rayleigh scattering by water vapor is neglected, since the effect is minor at temperatures below $120^{\circ} \mathrm{C}$ (Kopparapu et al. 2013). We do not include the effect of pressure broadening by water vapor on the molecular absorption coefficients. A discussion and a comparison between our model and the model used by Goldblatt et al. (2013) are presented in the appendix.

Clouds are incorporated in the radiation calculation, assuming the so-called maximum-random-overlap assumption; that is, cloud layers are assumed to be maximally overlapping if they are adjacent to one another and randomly overlapping if they are separated by a clear layer. Clouds are not assumed to be blackbodies; rather, their absorptivity depends on the combined optical depths of the cloud, the gas in which they are embedded, and the interstitial aerosol. The microphysics to determine the optical properties of the cloud particles involve the liquid water and ice paths and cloud drop radii, as well as liquid water and ice content. Cloud scattering is included in the form of a single-scattering albedo in the shortwave calculation but is neglected in the longwave calculations. The single-scattering albedo is calculated by assuming Mie scattering from cloud droplets. Clouds are not considered in the radiative transfer routines if the cloud condensate does not exceed $10^{-7} \mathrm{~kg}$ per $1 \mathrm{~kg}$ of air.

\section{2) HydrologicAl CYCLE}

Water vapor enters the atmosphere by means of evaporation at the surface. The water (in all phases) is then distributed within the column by convection and vertical diffusion. Water can leave the column by means of convective and large-scale precipitation and by surface condensation. The specific mass of water (in all phases) and, as a consequence, the relative humidity in the model are the result of this hydrological cycle. Precipitation is created by the convection scheme, as well as by the cloud-microphysical scheme. Even though the convection scheme provides information about detrained water to the cloud-microphysical scheme, cloud condensate is created or destroyed by the cloud microphysical scheme only.

\section{3) Convection}

ECHAM6 uses a mass flux scheme for cumulus convection (Tiedtke 1989), with modifications for penetrative convection according to Nordeng (1994). The contribution of cumulus convection to the large-scale budgets of heat, moisture, and momentum is represented by an ensemble of clouds consisting of updrafts and downdrafts in a steady state. Depending on moisture convergence at the surface and depth of the convection cell, the model will either run in penetrative, midlevel, or shallow convection mode.

\section{4) Clouds}

We make use of the Sundqvist scheme for fractional cloud cover (Sundqvist et al. 1989). This cloud scheme has been tuned for use with present-day Earth's climate (Lohmann and Roeckner 1996; Mauritsen et al. 2012). Obviously, we cannot ascertain that the choices of the tuning parameters are still valid for temperatures of up to $120^{\circ} \mathrm{C}$. However, the model relates cloud cover to relative humidity. Since relative humidity should be crucial to cloud formation irrespective of the SST, we expect the models not to go far astray at high temperatures. We also perform a few sensitivity experiments with another scheme for cloud cover (Tompkins 2002), which does not only relate cloud cover to relative humidity but explicitly models the subgrid variability of water.

The cloud microphysical scheme is the same for both cloud cover schemes and is described in detail in Lohmann and Roeckner (1996). However, the microphysical scheme is handled differently by the two cloud cover schemes in that the threshold for cloud growth is different. The cloud microphysical scheme consists of prognostic equations for the vapor, liquid, and ice phases. There are explicit microphysics for warm-phase, mixed-phase, and ice clouds. The cloud condensation nuclei concentration follows a prescribed vertical profile that is typical for present-day Earth maritime conditions. Since we have no estimate of the aerosol load in a hypothetical warm climate, we just assume that this profile is also a reasonable choice for warmer climates. The microphysics does not require changes for the use of the scheme in warm climates, since cloud formation in a warm-phase cloud, in which potential changes may occur, is not directly temperature dependent (at least not in the range of temperatures that we consider). Hence, the present formulation should be valid.

\section{(i) The Sundqvist scheme}

We do not discuss the parameterization of fractional cloud cover in detail but give a short summary thereof; for more details, see Lohmann and Roeckner (1996). Cloud cover $C$ is parameterized as a function of relative humidity $r$ at each layer of the column. For $r>r_{0}$, where $r_{0}<r_{\text {sat }}$ is a subgrid-scale condensation threshold and $r_{\text {sat }}$ is the relative humidity of saturation (=1 in general), condensational growth of cloud droplets occurs. The threshold $r_{0}$ is an empirical function of pressure and decreases from $r_{0}\left(p_{\text {surf }}\right)=0.9$ at the surface to $r_{0}(p \approx 500 \mathrm{hPa})=0.7$ and remains constant at 0.7 above about $500 \mathrm{hPa}$.

\section{(ii) The Tompkins scheme}

Again, we do not discuss the parameterization of fractional cloud cover in detail, but we give a short 
TABLE 1. Overview of the experimental setup.

\begin{tabular}{lll}
\hline \hline & \multicolumn{1}{c}{ CSC } & \multicolumn{1}{c}{ FSC } \\
\hline Applied TSIs (multiples of $\left.S_{0}\right)$ & & \\
$\quad$ Cold start & $0.95,1.00,1.10,1.15,1.20$ & $0.90,0.95,1.00,1.10,1.20,1.25,1.30,1.40,1.45$ \\
$\quad$ Warm start & $0.95,1.00,1.10,1.15,1.20$ & $0.90,0.95,1.00,1.10,1.20,1.30,1.40,1.45$ \\
Initial SSTs (K) & $287.8,288.8,289.8$ & $287.8,288.8,289.8$ \\
$\quad$ Cold start & $383.4,384.4,385.4$ & $383.4,384.4,385.4$ \\
$\quad$ Warm start & & 3 \\
\hline
\end{tabular}

summary thereof; for more details, see Roeckner et al. (2003). In simple terms, the difference between the Tompkins scheme and the Sundqvist scheme is that the Tompkins scheme does not only relate fractional cloud cover directly to relative humidity as the Sundqvist scheme does, but it also models the fluctuations of water within each layer of the column in order to determine the fractional cloud cover. Furthermore, the Tompkins scheme takes information directly from other processes of the hydrological cycle to calculate the cloud cover. In technical terms, the scheme is based on a statisticaldynamical approach. Neglecting temperature fluctuationsthat is, assuming that subgrid-scale cloud formation is caused solely by fluctuations in total water content- the fractional cloud cover can be expressed as the integral of a beta distribution. This beta distribution represents the probability density function of the abundance of water (in all phases). The choice of the probability density function was guided by simulations with a cloudresolving model, which was run on a horizontal domain of about $90 \mathrm{~km} \times 90 \mathrm{~km}$ and $21 \mathrm{~km}$ in the vertical (Tompkins 2002).

\section{b. Experiments}

Simulations are performed until either a steady state is attained or the SST exceeds $393.15 \mathrm{~K}$. Since for this SST the ocean exceeds the boiling temperature at normal pressure and hence water vapor becomes very abundant in the atmosphere, the atmosphere is opaque enough to shield effectively the radiation emitted from the surface. Hence, it is likely that the OLR decouples from the SST, in which case a runaway greenhouse would occur (Nakajima et al. 1992). However, we should keep in mind that our setup does not allow us to determine whether the oceans will completely evaporate or not. We will, nonetheless, speak of a runaway greenhouse if the simulations are terminated because of the temperature exceeding $393.15 \mathrm{~K}$.

The obliquity of our planet is set to zero, and we assume a perfectly spherical orbit around its star. Hence, there is no seasonal cycle. Since the velocity of rotation is not changed from present-day Earth, the column experiences a day-night cycle of $24 \mathrm{~h}$. The column is located at $38^{\circ} \mathrm{N}$, because in this case the temporal average of the angle of incidence of the solar radiation in the column corresponds to the temporal and spatial average of solar radiation on the sphere. The TSI is varied by changing the total intensity of the flux but leaving the ratio of intensities per band unchanged.

We perform all simulations for FSC as well as for CSC. Clouds are transparent to radiation in CSC, but all the other cloud physics are active. All the physics are active in FSC, including the cloud-radiation interaction. To obtain comparable settings for CSC and FSC, we increase the surface albedo in CSC such that the temporal average of SST does not differ by more than $1 \mathrm{~K}$ from the value obtained for FSC when the present-day value of TSI is applied. The surface albedo is therefore increased from 0.07 to 0.22 in CSC. The greenhouse gas concentrations are kept at present-day values (except for water vapor, which is a prognostic variable).

We denote the present-day value of TSI with $S_{0}$. The annually averaged incoming solar radiation $\overline{S_{0}}$ of the control simulation is $340 \mathrm{~W} \mathrm{~m}^{-2}$. As for a sphere, $\overline{S_{0}}$ corresponds to TSI/4 for the column. We conduct simulations for a combination of different initial conditions and of different TSIs for the two different setups. The applied TSIs of the simulations are listed in Table 1 for both CSC and FSC. For both setups, at least the largestapplied TSI leads to a runaway greenhouse.

For each value of TSI, six different initial conditions are considered, which are divided into two ensembles with different ranges of initial SSTs. The initial SSTs of the ensemble with a cold start are 287.8, 288.8, and $289.8 \mathrm{~K}$; and the atmospheric initial conditions are identical for all three SSTs. The initial SSTs of the ensemble with a warm start are $383.4,384.4$, and $385.4 \mathrm{~K}$, again with identical atmospheric initial conditions for all three SSTs for CSC and FSC. The reason for choosing two ensembles with a very different range of initial SST is that we want to investigate whether multiple equilibria are possible (Rennó 1997). Note that our approach does not allow us to determine whether we find all possible equilibria. 

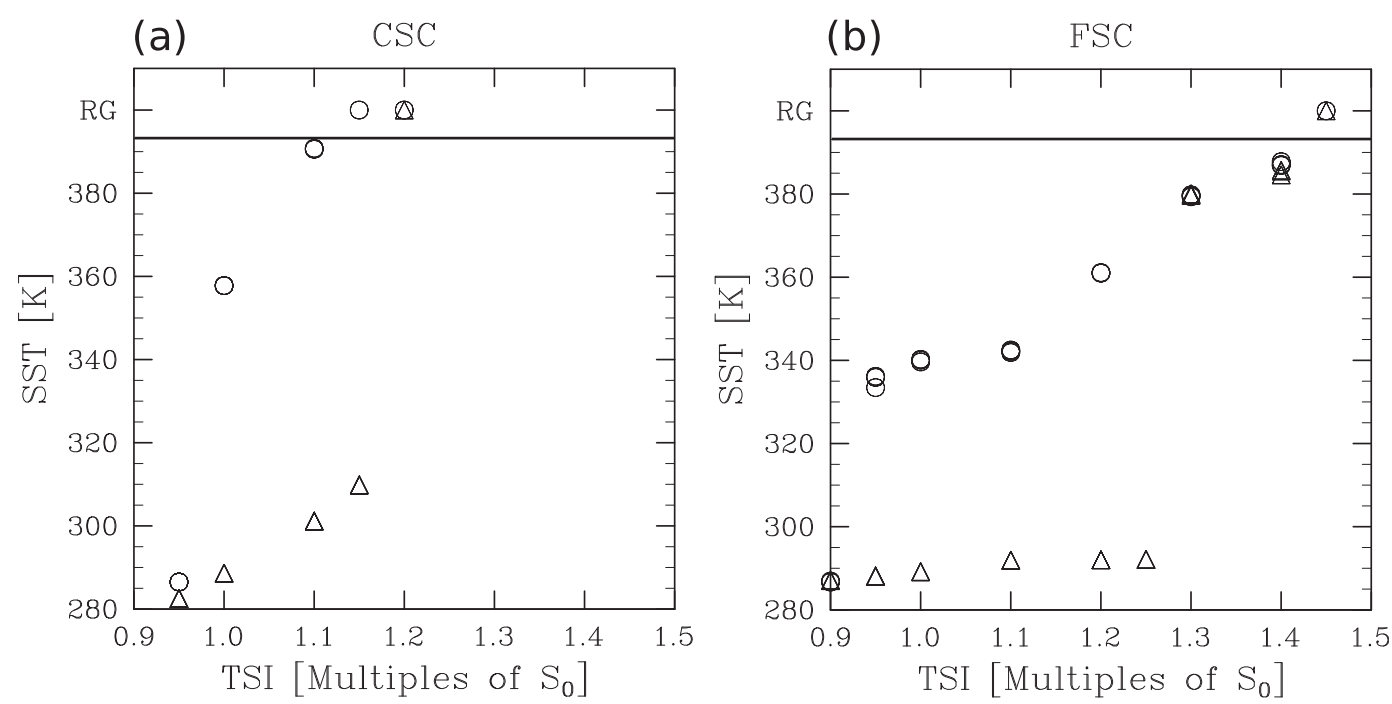

FIG. 1. Temporal mean of the SST over the last 5 years of the simulation as a function of TSI for (a) CSC and (b) FSC. Triangles indicate simulations with a cold start, and circles denote simulations with a warm start. Marks located above the horizontal line denote simulations in which a runaway greenhouse (RG) occurs.

\section{Results}

\section{a. Critical TSI and multiple equilibria}

\section{1) Clear-SKy CONDITIONS}

For CSC, a runaway greenhouse occurs for all simulations with TSIs larger than $1.15 S_{0}$ (Fig. 1). For a TSI of $1.15 S_{0}$, all simulations with a cold start attain an equilibrium with SSTs of around $310 \mathrm{~K}$, whereas all simulations with a warm start go into a runaway greenhouse. Hence, the critical value of TSI to trigger a runaway greenhouse for a warm start is smaller than for a cold start. Two different equilibria are attained for a TSI of $1.00 S_{0}$ and $1.10 S_{0}$, with temperatures below $305 \mathrm{~K}$ for simulations with a cold start and temperatures above $350 \mathrm{~K}$ for those with warm start; this result is consistent with Rennó (1997). The simulations with a warm start for TSIs of $0.95 S_{0}$ end up in the cold regime. Therefore, the lower limit for double equilibria lies in the range of $0.95-1.00 S_{0}$.

\section{2) FULL-SKY CONDITIONS}

For FSC a runaway greenhouse occurs for all simulations with a TSI of $1.45 S_{0}$ (Fig. 1). All simulations attain steady states with SSTs of approximately $385 \mathrm{~K}$ for TSIs between $1.30 S_{0}$ and $1.40 S_{0}$; hence, unlike for CSC, the simulations with a cold start can attain a steady state at high SSTs. Warm and cold starts result in different equilibria, with SSTs above $335 \mathrm{~K}$ for a warm start and below $295 \mathrm{~K}$ for a cold start for TSIs between $0.95 S_{0}$ and $1.25 S_{0}$, indicating a bistability in the climate. All simulations with a TSI of
$0.90 S_{0}$ attain an equilibrium in the cold regime; thus, the lower limit for bistability lies between $0.90 S_{0}$ and $0.95 S_{0}$.

\section{$b$. The effect of clouds on the energy balance}

In the previous section, we showed that clouds increase the TSI needed to trigger a runaway greenhouse substantially. This result is not trivial, because both shortwave and longwave cloud feedbacks could also be positive. The combined feedback could thus lead to a critical TSI to trigger a runaway greenhouse that is smaller for FSC than for CSC. Therefore, we show in this section how the clouds affect the energy balance.

\section{1) FULL-SKY CONDITIONS FOR PRESENT-DAY TSI}

To facilitate the interpretation of our results, we present the cloud climatology for present-day simulations for FSC (Fig. 2). We have a slightly larger effective albedo than for present-day Earth: we find 0.32. The cloud albedo is more strongly influenced by the lower clouds at the top of the boundary layer than by the clouds in the upper troposphere despite the cloud cover being larger for the upper clouds, because the lower clouds contain more condensate. To maintain the energy balance, the OLR is therefore lower than for present-day Earth, with values of $231 \mathrm{~W} \mathrm{~m}^{-2}$. We choose not to tune our model to fit Earth's cloud climatology perfectly but to keep the values of the tuning parameters in the range that gives the best results in threedimensional simulations (Mauritsen et al. 2012). Thus, we ascertain that the interaction between dynamics and 

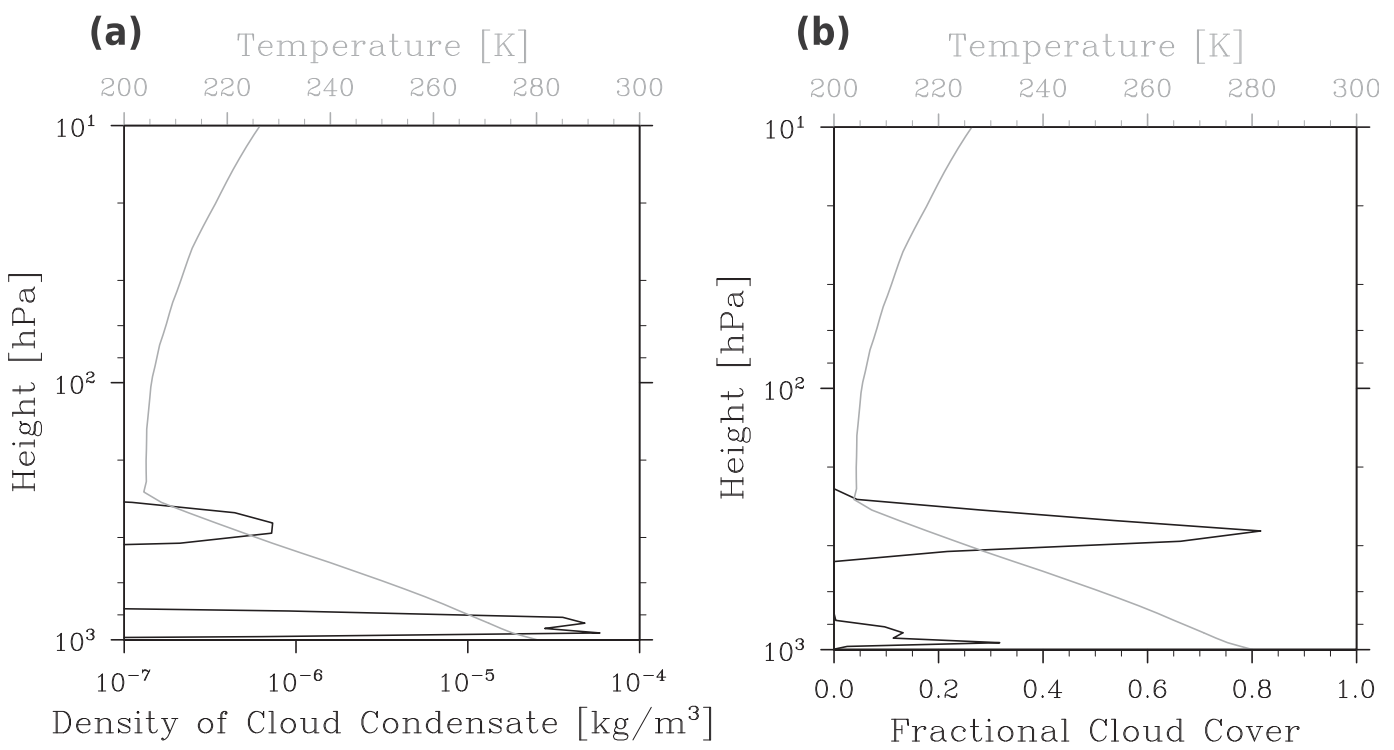

FIG. 2. Atmospheric profiles of the temporal mean over the last 5 years of simulation of (a) cloud condensate (black) and temperature (gray) and (b) cloud cover (black) and temperature (gray). The vertical axis is the height in terms of pressure of dry air. The panels show the results of one simulation with a cold start for present-day insolation with FSC. This is the control simulation for present-day climate.

cloud formation is well represented for present-day conditions. Because of the inherently simplified geometry of a one-dimensional model compared to a threedimensional model, it is not surprising that our onedimensional model does not reproduce Earth's averaged energy balance exactly. We will keep this in mind when discussing the results.

\section{2) TRANSIENT BEHAVIOR OF THE CLOUd PROPERTIES}

Between $1.00 S_{0}$ and $1.25 S_{0}$ the equilibrium SST is increasing only weakly with increasing TSI, because in equilibrium the effective albedo increases as well with increasing TSI (Fig. 3). The increase in albedo is caused (a)

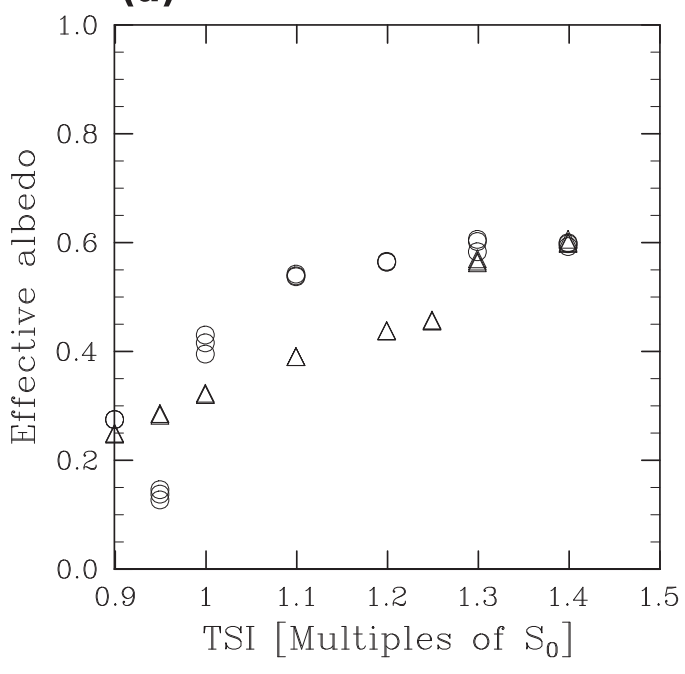

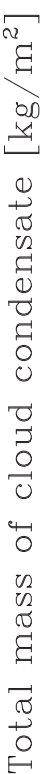

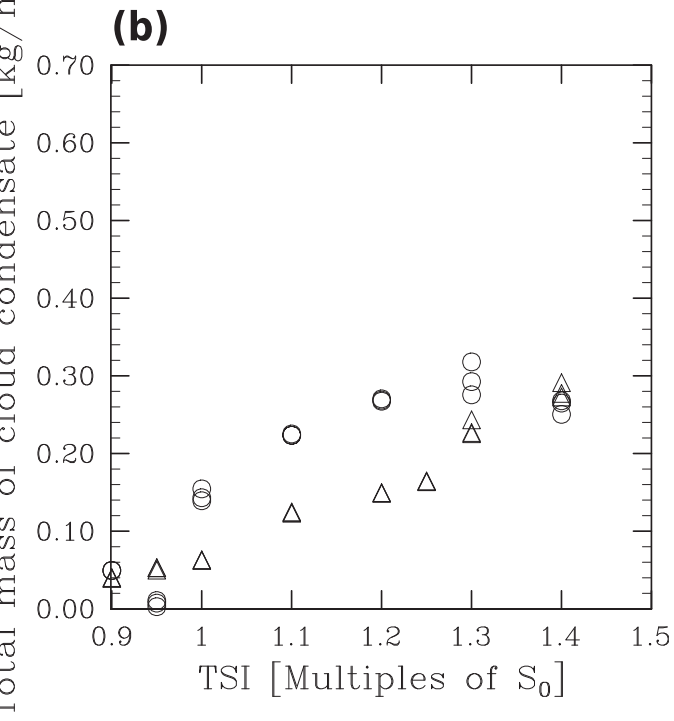

FIG. 3. Temporal mean over the last 5 years of simulation of the (a) effective albedo and (b) vertically integrated cloud condensate as a function of TSI for FSC. Triangles indicate simulations with a cold start, and circles denote simulations with a warm start. Squares denote the simulations with a warm start that were performed with the fixed condensation algorithm. There are no marks for simulations in which a runaway greenhouse occurs. 
(a) Density of Cloud Condensate $\left[\mathrm{kg} / \mathrm{m}^{3}\right]$

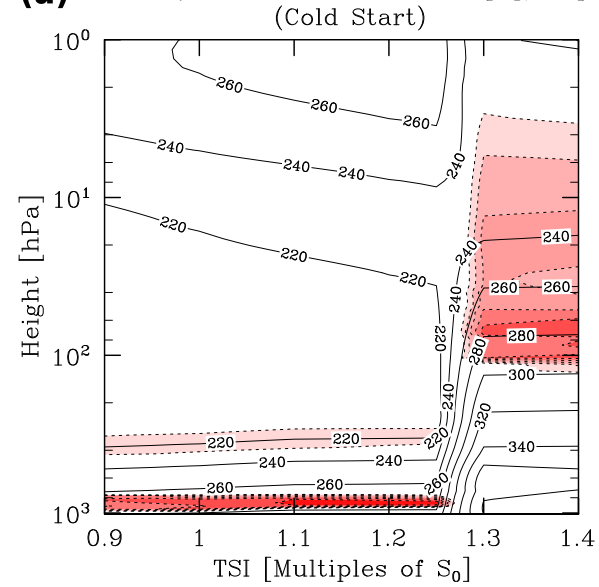

(c) Fractional Cloud Cover

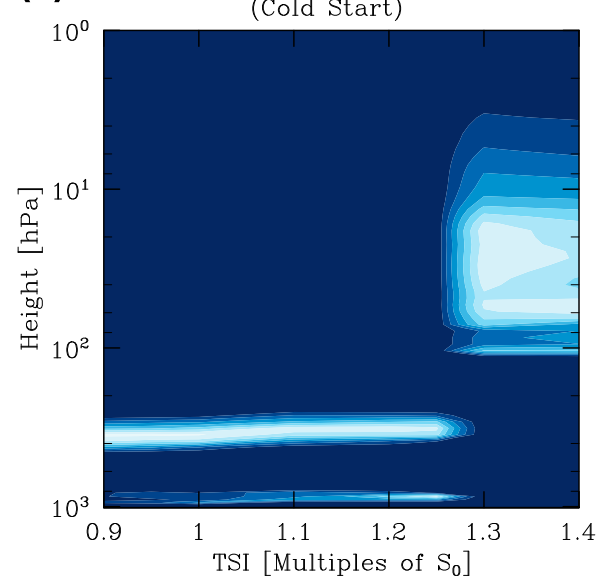

(b) Density of Cloud Condensate $\left[\mathrm{kg} / \mathrm{m}^{3}\right]$

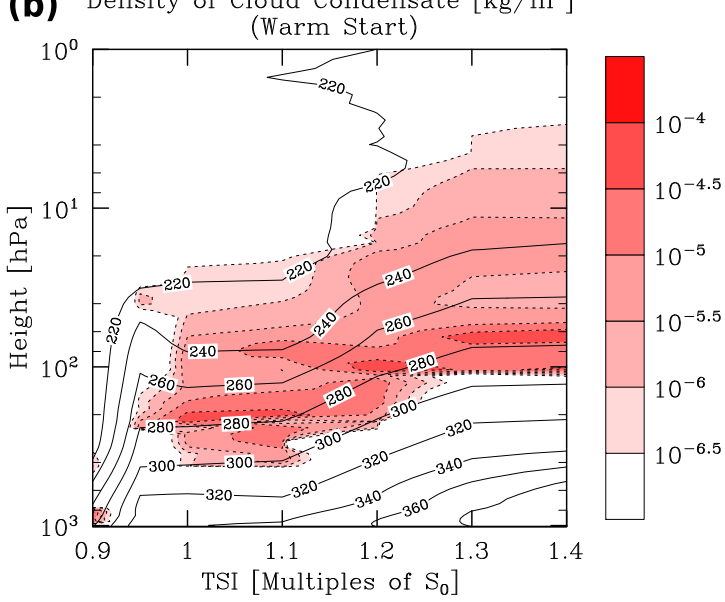

(d) Fractional Cloud Cover (Warm Start)

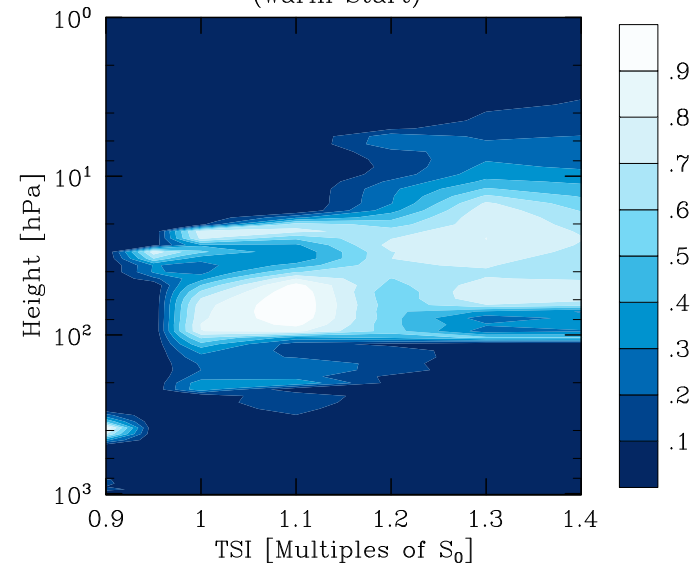

FIG. 4. The temporal mean over the last 5 years of simulation of (a) cloud condensate and (c) fractional cloud cover of one ensemble member with a cold start for different values of TSI. (b),(d) As in (a),(c), but for one of the ensemble members with a warm start. The vertical coordinate is height (in terms of pressure of dry air). The line contours in (a),(c) denote the temperatures with a $20-\mathrm{K}$ spacing between lines.

by an increase of both fractional cloud cover and cloud condensate of the water clouds in the lower troposphere (Fig. 4). The low clouds form at the top of the boundary layer, which is, however, not recognizable in Fig. 4 because of the coarse temperature resolution of the contours. Clouds remain approximately at the same position in the cold regime, but the temperature within the boundary layer increases gently with TSI. This increase in temperature leads to an increase in moisture within the boundary layer (not shown), which explains the increased amount of cloud condensate within the clouds. However, as a critical temperature is exceeded, a further increase in SST leads to a decrease of relative humidity at the top of the boundary layer (not shown), which causes the clouds at the top of the boundary layer to vanish (Fig. 5). The disappearance of the boundary layer clouds and the associated drop in albedo are the cause of the climate transition toward high temperatures. Only after the atmospheric structure has substantially changed is a new equilibrium attained, because eventually the shortwave effect outweighs the longwave effect of the clouds that form in the upper troposphere in the warm state (Fig. 5). Clouds form at temperatures below $300 \mathrm{~K}$ in this warm regime (Fig. 4). The spatial extent of the cloud region is relatively wide and reaches up to where the temperature falls to $230 \mathrm{~K}$. The largest density of cloud condensate occurs at a temperature of around $280 \mathrm{~K}$ in the warm state (Fig. 4), but the density of cloud condensate drops more slowly with temperature than in the cold regime. As a consequence, the OLR is smaller (around $200 \mathrm{~W} \mathrm{~m}^{-2}$ on average) in this warm state compared to the cold state. The total amount of cloud condensate is larger, which tends to make the clouds more reflective (Fig. 3). Furthermore, the cloud 
(a)

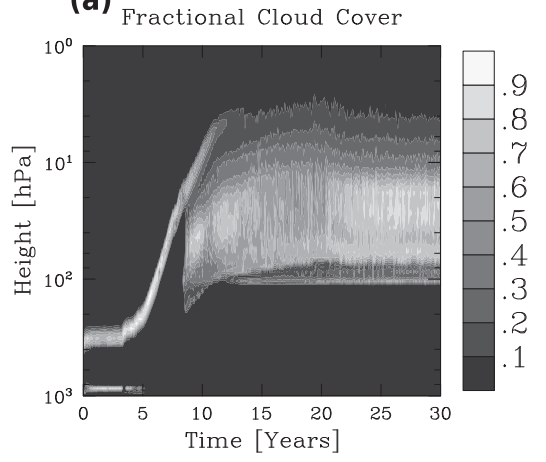

(b)

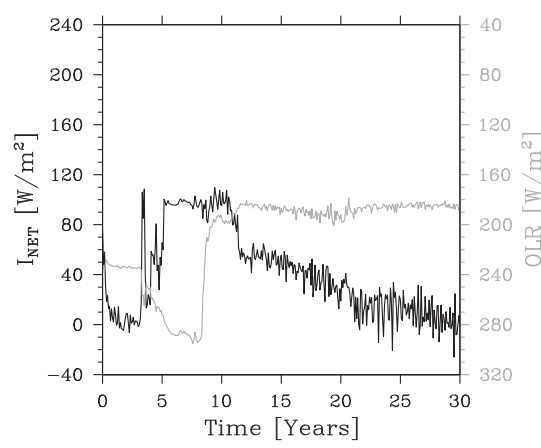

(c)

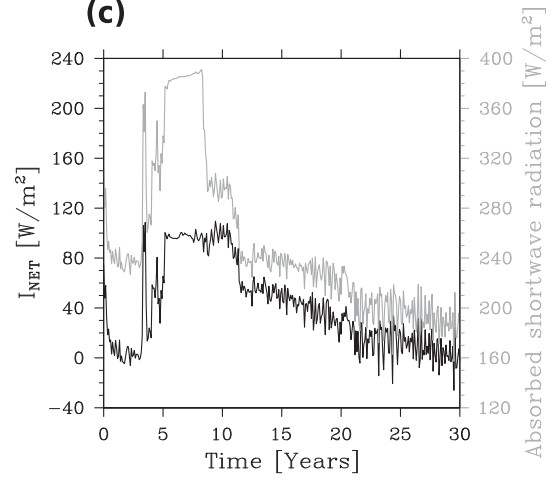

FIG. 5. (a) The temporal evolution during the transient period of fractional cloud cover as a function of time and height (in terms of pressure of dry air) for a simulation with a cold start for a TSI of $1.30 S_{0}$ for FSC. (b) The temporal evolution of the radiative imbalance at the top of the atmosphere $I_{\text {NET }}$ (black line) against the OLR (gray line) for the same simulation. Note that the axis for the OLR has been flipped, since a decrease of OLR leads to an increase of $I_{\text {NET }}$. (c) The temporal evolution of $I_{\mathrm{NET}}$ (black line) against the total absorbed shortwave radiation by the surface and the atmosphere (gray line) for the same simulation. Note that $I_{\mathrm{NET}}$ is equal to the difference of the total absorbed shortwave radiation and the OLR.

condensate is distributed over a relatively large vertical extent; as a consequence, the cloud droplets remain relatively small, which increases the albedo of the clouds even further.

All simulations with a TSI larger than or equal to $0.95 S_{0}$ and a warm start terminate in the warm regime. The clouds in these simulations are very similar to the ones with a cold start that end up in the warm regime, except that both cloud cover and cloud condensate decrease with decreasing TSI (Fig. 4). The clouds form in the same range of temperatures in all these simulations. For TSIs below $1.1 S_{0}$, both cloud cover and cloud condensate decrease strongly with TSI until nearly no cloud condensate is left for a TSI of $0.95 S_{0}$. A further reduction of TSI to $0.90 S_{0}$ then leads to a transition to the cold regime.

\section{c. Summary}

It stands out that the critical TSI to trigger a runaway greenhouse is markedly higher for FSC than for CSC. The critical TSI is in the range of $1.15-1.20 S_{0}$ for CSC with initial SSTs from 287.8 to $289.8 \mathrm{~K}$ and $1.10-1.15 S_{0}$ for initial SSTs from 383.4 to $385.4 \mathrm{~K}$. The critical TSI for FSC is in the range of $1.40-1.45 S_{0}$, with multiple equilibria occurring for a wide range of TSI (from 0.90$0.95 S_{0}$ to $1.25-1.30 S_{0}$ ).

The simulations with FSC show that it is exclusively the increased cloud albedo that prevents a runaway greenhouse from occurring for large values of TSI. Clouds become increasingly optically thick with increasing TSI, which causes the cloud albedo to increase as well. In the warm regime, clouds occur in a welldefined range of atmospheric temperature in the upper troposphere, irrespective of the TSI. The climate transition from the cold to the warm climate is caused by the disappearance of clouds at the top of the boundary layer, which causes a temporary drop in the albedo.

\section{Discussion}

\section{a. Boundary layer clouds}

Both fractional cloud cover and cloud condensate at the top of the boundary layer first increase with TSI (Fig. 4). As a critical TSI is attained, both cloud cover and cloud condensate at the top of the boundary layer decrease and eventually vanish (Fig. 5). We discuss here why our model exhibits this behavior and compare our results to the literature.

The moisture content in the boundary layer increases with the SST. The relative humidity at the top of the boundary layer, the fractional cloud cover, and the amount of cloud condensate increase as well. The increase in moisture content (and temperature) causes the moist static energy to increase faster in the boundary layer than in the layers above. Hence, the stability across the boundary layer decreases, and convection exports moisture out of the boundary layer with increasing intensity, which causes the relative humidity at the top of the boundary layer to decrease. This in turn eventually leads to the disappearance of the clouds. As the albedo drops and strong surface heating occurs, the convection becomes strong enough to temporarily destroy the boundary layer. This mechanism explains not only the behavior of clouds in our model but also the dependence of the disappearance of the clouds on the temperature (the clouds start disappearing at SSTs of approximately $293 \mathrm{~K}$ ), since the moist static energy depends on moisture 

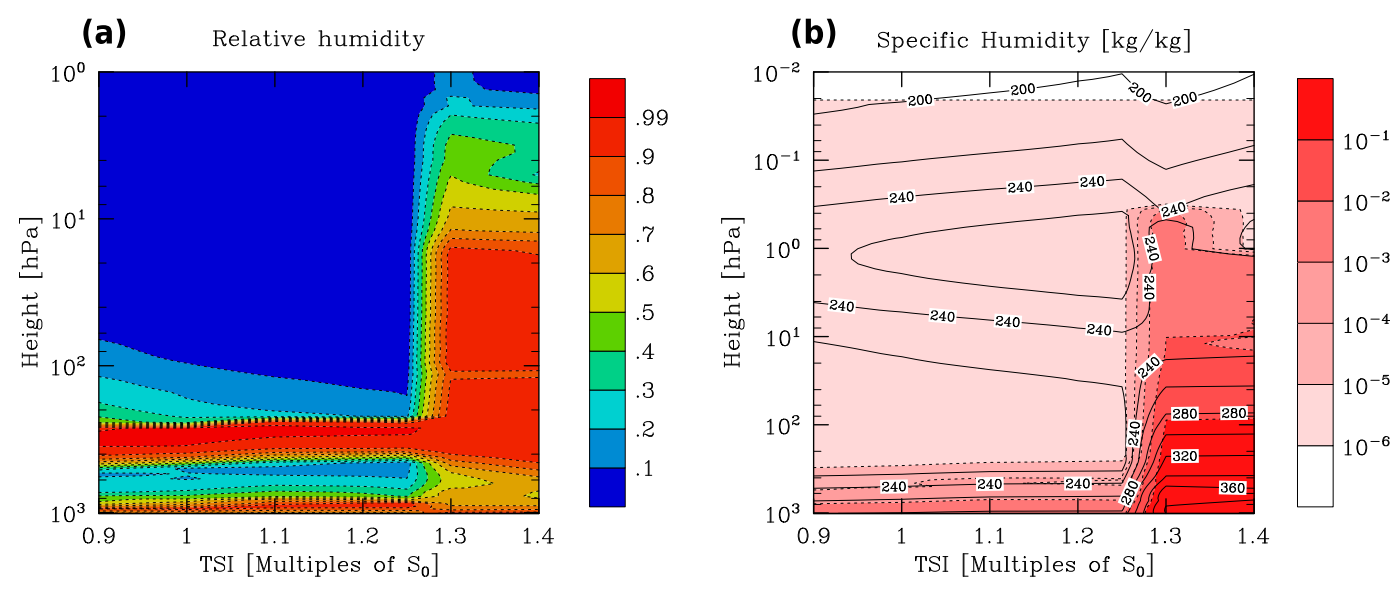

FIG. 6. The temporal mean over the last 5 years of simulation of (a) the relative humidity and (b) the specific humidity of one ensemble member with a cold start for different values of TSI for FSC. The vertical coordinate is height (in terms of pressure of dry air). Note that the relative humidity is plotted up to $1 \mathrm{hPa}$, whereas specific humidity is plotted up to $10^{-2} \mathrm{hPa}$ (i.e., the top level). The line contours in (b) denote the temperatures with a $20-\mathrm{K}$ spacing.

and, hence, on temperature rather than on the applied forcing.

Our boundary layer clouds can best be compared to boundary layer clouds at low latitudes, since the largescale motions are less important there than at higher latitudes. Model simulations have shown both an increase and a decrease of low clouds at low latitudes with increasing temperature (Bony et al. 2006), but Clement et al. (2009) infer from the analysis of observations and model results of the northeastern Pacific that the low-latitude low-level clouds decrease with increasing temperatures. We cannot, however, conclude that the mechanisms at work would lead to the complete disappearance of boundary layer clouds. Brient and Bony (2013) suggest that the decrease in low-level clouds with increasing temperature produced by the L'Institut Pierre-Simon Laplace Coupled Model, version 5A (IPSL-CM5A) is triggered by a decrease of the vertical gradient of moist static energy, which leads to dry air being mixed into the boundary layer in low-latitude subsidence regions and hence to a drying. The decrease of the gradient of moist static energy also causes the cover of low clouds to decrease in our model, but the mechanism for the decrease is different, since our onedimensional model cannot simulate the drying of the boundary layer in the subsidence regions. If the process of Brient and Bony (2013) is indeed responsible for a decrease in low-level clouds, then this decrease should not occur as abruptly as in our model. The simulations of Boer et al. (2005) show only little changes in cloud cover and albedo for a TSI of up to $1.15 S_{0}$, but both total cloud cover and albedo decrease in the simulation with $1.25 S_{0}$. However, their fractional cover of boundary layer clouds does not change substantially.
The value of TSI necessary to make the boundary layer clouds disappear is large (around 1.25-1.30 $S_{0}$ ). However, the annual average of net solar irradiance on present-day Earth at the equator is more than $20 \%$ larger than the global average. Therefore, our results suggest that a TSI of around $1.05 S_{0}$ could be sufficient to lead to the disappearance of the boundary layer clouds at the equator. Such an extrapolation is obviously too simplistic, but it serves as a reminder that low-level boundary layer clouds on an Earthlike planet may disappear at a lower TSI than our results may at first suggest. This has recently been confirmed by a threedimensional study (Leconte et al. 2013).

\section{b. Humidity profiles}

The humidity structure of the atmosphere is shown in Fig. 6. There are two aspects that we discuss here, which are the high tropospheric relative humidity in the warm regime and the structure of moisture in the upper atmosphere in the warm regime.

The relative humidity in the troposphere is considerably higher in the warm regime than in the cold regime (Fig. 6). This can be explained with the argument by Rennó (1997) that the relative humidity in warm, and hence optically thick, regions of the atmosphere should be high, because radiative cooling can hardly balance the heating that would be induced by an unsaturated downdraft. However, even though the tropospheric relative humidity is higher in the warm regime, most of the troposphere is not permanently saturated. This result refutes the recent argument by Wolf and Toon (2014) and Leconte et al. (2013) that unsaturated regions cannot be represented in a one-dimensional model. The relative humidity at the surface is also larger in the warm regime 
(above 0.9) compared to the cold regime (between 0.6 and 0.75). According to Kasting et al. (2014), this is expected, because the latent heat flux would become too large to be balanced by the other surface fluxes if the relative humidity remained constant with increasing SST. So, in general, the high tropospheric relative humidity we find in the warm regime appears to be plausible.

Even though most of the middle atmosphere becomes considerably moister with increasing SSTs, the top levels of our model remain very dry (Fig. 6). This is in contradiction to atmospheres modeled by a moist adiabatic lapse rate used in several previous one-dimensional studies, where the specific humidity at the top of the atmosphere strongly increases with SST (e.g., Kasting 1988; Kopparapu et al. 2013; Goldblatt et al. 2013). There are several reasons for this difference. The tropopause climbs less in our study, because temperature decreases faster with height in our model since condensation heat is not released to the same extent as in the case of strictly moist adiabatic ascent. Therefore, the mixing rate of water vapor at the tropopause in our model is smaller than for the aforementioned studies. Furthermore, our atmosphere contains ozone, implying that the upper atmosphere is more stably stratified. Therefore, mixing of water vapor by diffusion into the stably stratified upper atmosphere is slow, and the regions of the atmosphere above the tropopause are considerably drier than the tropopause itself. However, even though we limit tropospheric ozone concentrations, a chemistry model would be required to calculate the effect of increased water vapor concentrations on the ozone consistently. The slow mixing within the stratosphere also causes another effect: in the simulations with a warm start and a TSI sufficiently low for the simulations to return into the cold regime, the specific humidity in the middle atmosphere remains considerably larger when the cold steady state is attained than in simulations that started in the cold regime. This occurs because, as the column cools, the tropopause falls to lower levels, leaving considerable amounts of water vapor in the now stably stratified levels where the specific humidity decreases only very slowly with time. The difference in stratospheric humidity between simulations with a warm start and those with a cold start (both of which end in the cold regime) also explains the small differences in the energetics for simulations with the same TSI but different initial conditions. We expect that in the presence of three-dimensional dynamics such as the Brewer-Dobson circulation, the stratospheric mixing would be much more efficient; therefore, the effects discussed in this paragraph may not be seen in threedimensional simulations.

\section{c. Clouds in the warm regime}

In this section, we explain why clouds have a strong cooling effect in the warm regime and why clouds do not form in some regions of the lower troposphere despite a high relative humidity.

\section{1) THE IMPACT OF THE SHORTWAVE AND THE LONGWAVE EFFECT OF CLOUDS IN THE WARM REGIME}

The shortwave effect of clouds dominates their longwave effect, as thick clouds form in the warm regime for two reasons. First, the longwave absorption saturates faster with the mass of cloud condensate than does the cloud albedo (Pierrehumbert 2010). If there is enough cloud condensate to make a cloud act like a blackbody, then a thickening of the cloud has no further effect on the OLR, but the reflected shortwave radiation would still increase. The clouds contain sufficient condensate in the warm regime to act as a blackbody in all our simulations.

Second, there is a lower limit to the emission temperature of clouds. This is important because the longwave cloud radiative effect could outweigh the shortwave cloud radiative effect if optically thick clouds were to form at ever-lower temperatures, which would cause the emission temperature to decrease as well. But since the mass of possible condensate depends on temperature through the Clausius-Clapeyron relation, clouds become optically thin below a certain temperature, and the emission temperature of clouds has a lower bound. We find very little cloud condensate below $220 \mathrm{~K}$, and the emission temperature does not fall below $235 \mathrm{~K}$ and varies less with SST in the warm regime than in the cold regime.

\section{2) SUPPRESSION OF CLOUD FORMATION IN THE OPTICALLY THICK REGION OF THE ATMOSPHERE}

For equilibrium SSTs in the warm regime, the relative humidity exceeds the threshold value for cloud formation in most of the troposphere. Nonetheless, cloud condensate never forms where the water vapor density exceeds $0.05 \mathrm{~kg} \mathrm{~m}^{-3}$. This is explained by analogy with Rennó (1997), who argued that an optically thick region of the atmosphere should be close to saturation. In this region, the radiative cooling tends to zero because of the high opacity. In equilibrium, the convective downdraft must therefore be saturated to compensate for the saturated convective updraft. This argument carries over to condensation: In the regions of the atmosphere where, because of high opacity, radiative cooling is ineffective, condensation heat cannot be effectively dissipated. Therefore, the condensation heat will cause reevaporation 

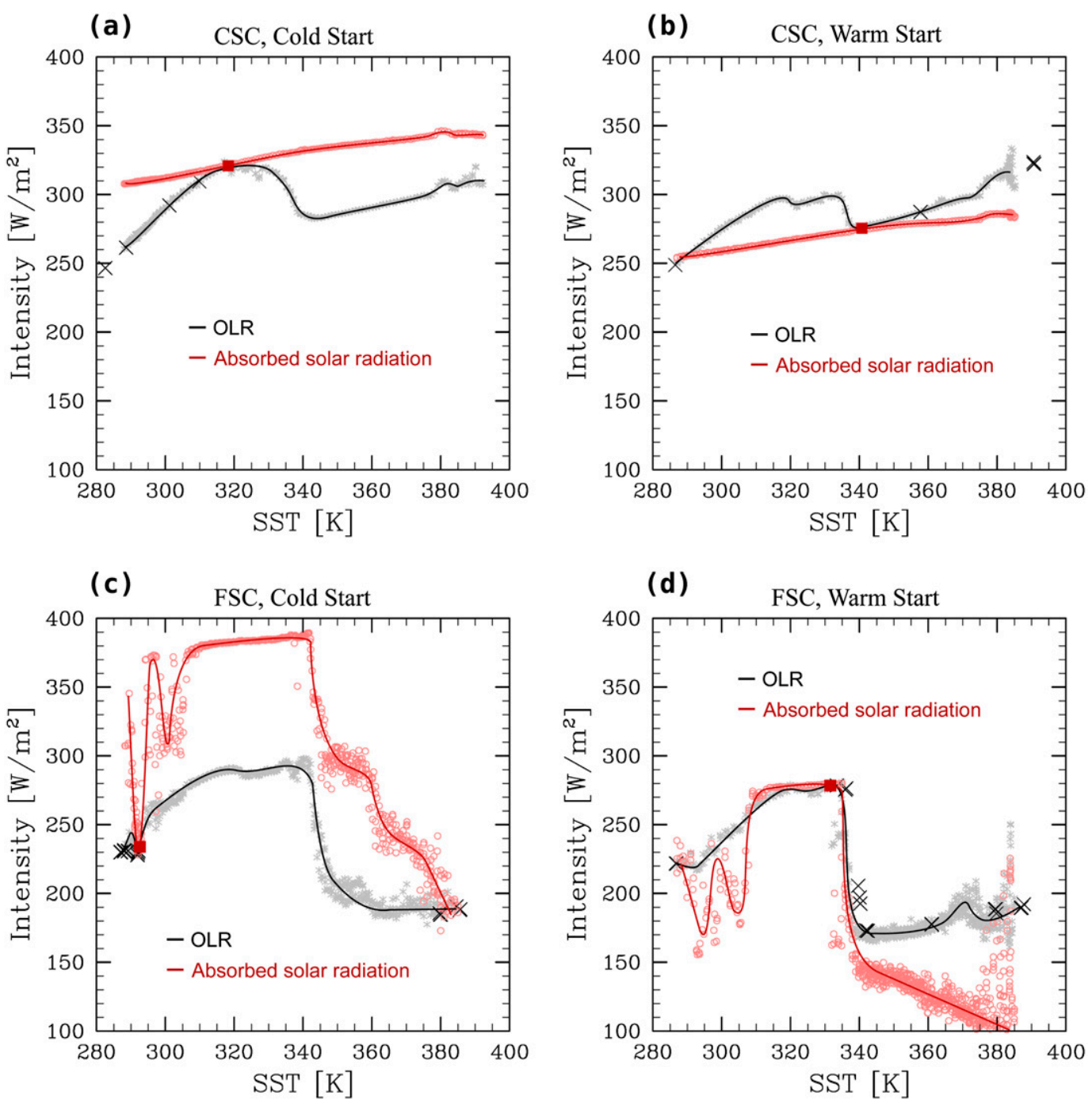

FIG. 7. Synthesis of OLR and of the net absorbed solar radiation as a function of SST for (a),(c) simulations with a cold start and (b),(d) simulations with a warm start for (a),(b) CSC and (c),(d) FSC. The large black crosses denote the OLR in steady state for the various setups. Gray stars denote values of monthly means of the OLR taken from transient simulations at a spacing of $1 \mathrm{~K}$ for the specified setup (CSC or FSC and cold or warm start). The black line is a manual fit to the gray stars. The red circles are obtained by multiplying values of the albedo from transient simulations with a fixed value of TSI and, hence, approximate the total absorbed solar radiation for these values of TSI. The TSIs are $1.18 S_{0}$ for CSC with a cold start, $0.97 S_{0}$ for CSC with a warm start, $1.29 S_{0}$ for FSC with a cold start, and $0.94 S_{0}$ for FSC with a warm start. For the simulations with a cold start, these values of TSI are around the largest that still allow for a stable steady state in the cold regime. For the simulations with a warm start, these values of TSI are around the smallest that still allow for a steady state in the warm regime. The locations of these steady states are denoted by red squares.

of the condensate and hence suppress cloud formation. However, we cannot establish whether this effect is the only cause for the suppression of cloud formation.

\section{d. Multiple equilibria}

In both CSC and FSC, we find two stable steady states for a certain range of TSI. In this section, we explain why this is possible in each case and why, for certain ranges of
SSTs, no stable steady states are found. Most of the following arguments are based on the results shown in Fig. 7; therefore, we will first shortly illustrate how to analyze the figure. The figure shows the OLR and the absorbed solar radiation as a function of SST for four different setups. Steady states for the applied TSIs exist when the corresponding curve of the absorbed solar radiation intersects with the curve of the OLR. If the 
absorbed solar radiation is larger than the OLR at a particular SST, the column takes up energy and warms, whereas the column cools if the OLR is larger than the absorbed solar radiation. Therefore, a steady state is stable if the slope of the curve of the OLR is larger than that of the absorbed solar radiation at the location where the two curves intersect. If the slope of the OLR is smaller than the slope of the absorbed solar radiation where the two curves intersect, the steady state is unstable. Shifting the curve for the absorbed solar radiation upward and downward approximates small variations of TSI quite well because the effective albedo depends only weakly on TSI. Therefore, a simple way to estimate from Fig. 7 whether a steady state exists for a particular SST is to shift the curve for the absorbed solar radiation upward and downward and to check whether, at the given SST, the curve can intersect with the curve for the OLR.

\section{1) $\mathrm{CSC}$}

For CSC the absorbed solar radiation increases with SST (Figs. 7a,b), because the solar absorption by water vapor increases with increasing SST. Since stable steady states can only exist if the slope of the OLR is larger than the one of the absorbed solar radiation, stable steady states can thus only occur in CSC if the OLR increases with SST as well. There are two regimes where this is satisfied: one at SSTs below $320 \mathrm{~K}$ and one at SSTs above $340 \mathrm{~K}$. In both of these regimes, the slope of the OLR is larger than the one with the absorbed solar radiation, except close to the two local extremes (local maximum at $320 \mathrm{~K}$ and local minimum at $340 \mathrm{~K}$ ); therefore, stable steady states can potentially exist at nearly all SSTs in the two regimes. Furthermore, it can be inferred from Figs. $7 \mathrm{a}$ and $7 \mathrm{~b}$ that whenever a particular value of TSI allows for the curve of absorbed solar radiation to intersect with the OLR at SSTs above $340 \mathrm{~K}$, the same curve of absorbed solar radiation also intersects with the curve of the OLR at SSTs below $320 \mathrm{~K}$.

Now that we have diagnosed from an energetic point of view why double equilibria exist, we are going to explain the main physical mechanisms leading to their emergence. There are two crucial aspects that allow for the double equilibria: first, the decrease of OLR with increasing SST between 320 and $340 \mathrm{~K}$ and, second, the increase thereafter. The decrease of OLR with increasing SST allows for the OLR to assume the same value at different SSTs, and the increase of OLR at higher SSTs allows for the existence of the second regime of stable steady states. The decrease of OLR between 320 and $340 \mathrm{~K}$ is caused by a strong increase of tropospheric relative humidity (not shown), which leads

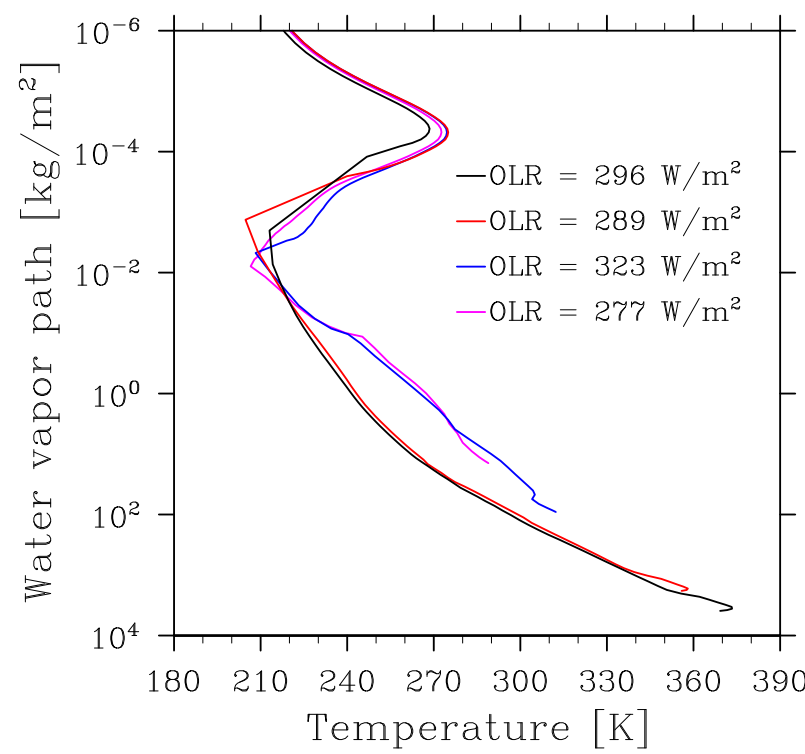

FIG. 8. Temperature as a function of the vertically integrated water vapor path from the top of the atmosphere for four different SSTs for one of the simulations with a cold start and a TSI of $1.20 S_{0}$. The water vapor path represents a simple measure for the clear-sky optical depth. For each of the profiles, the resulting OLR is specified. The four profiles have been chosen such that the change in atmospheric structure leading to a decrease in OLR with increasing SST is highlighted (Fig. 7a). The slopes of the magenta and the blue profiles are typical of the cold regime below $320 \mathrm{~K}$. The slopes of the red and the black profiles are typical of the warm regime above $340 \mathrm{~K}$. The OLR corresponding to the red profile is considerably smaller than the OLR corresponding to the blue profile, even though the surface temperature of the red profile is larger. This occurs because, in the range where the water vapor path is larger than $0.1 \mathrm{~kg} \mathrm{~m}^{-2}$, the temperature at any fixed water vapor path is considerably lower for the red profile than for the blue profile. As a consequence, the emission temperature is lower, and, hence, the OLR is smaller for the red profile.

to a large increase in atmospheric opacity and to a change in lapse rate (Fig. 8). Once this transition to high relative humidity is completed (at around $340 \mathrm{~K}$ ) the OLR attains a local minimum. The increase of OLR with SST thereafter occurs because the atmosphere is not yet sufficiently opaque at $340 \mathrm{~K}$ for the OLR to decouple completely from the SST. These two aspects leading to double equilibria are consistent with the ones found and explained by Rennó (1997) and by Pujol and North (2002). Unlike in Rennó (1997), our asymptotic value of OLR is most likely smaller than the local maximum that is attained at $320 \mathrm{~K}$; hence, as the TSI is increased, a transition from the cold regime to the warm regime is not possible, and the column goes directly into a runaway greenhouse (Fig. 7a). Therefore, for a cold start, the condition for a runaway greenhouse to occur is independent of the existence of steady states in the warm regime. 
(a)

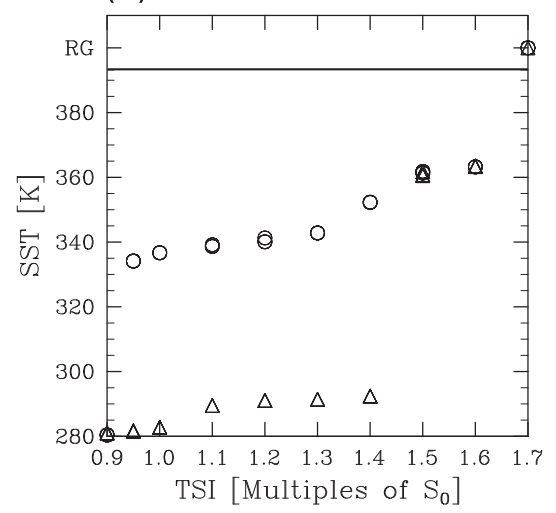

(b)

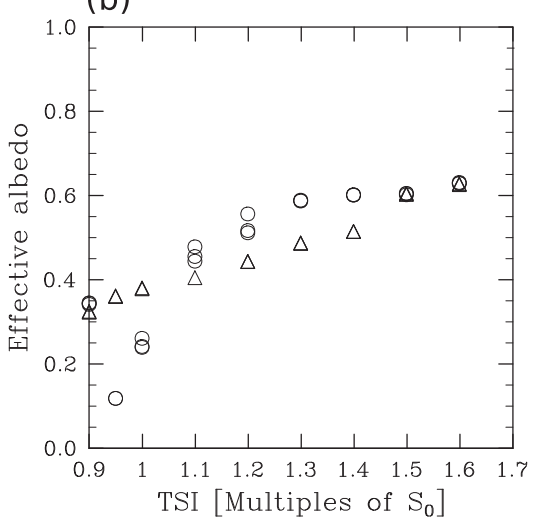

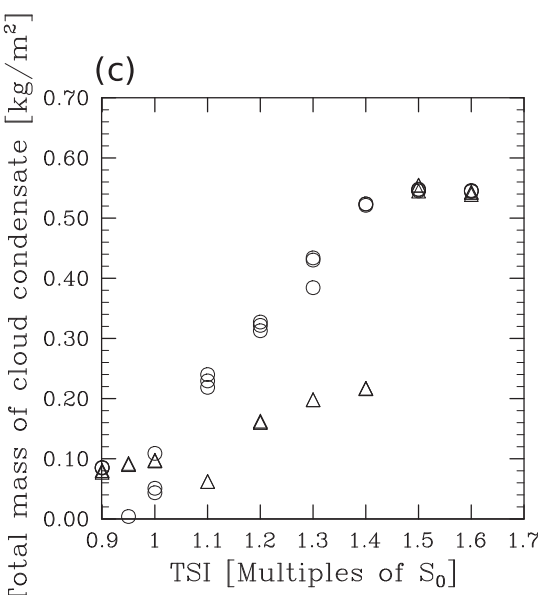

FIG. 9. Results from the simulations with the Tompkins scheme: Temporal mean over the last 5 years of simulation of (a) the SST, (b) the effective albedo, and (c) the vertically integrated cloud condensate with the Tompkins scheme as a function of TSI (the corresponding figures for the Sundqvist scheme are Figs. 1b and 3). Triangles indicate simulation with a cold start, and circles indicate simulations with a warm start. Marks located at RG above the horizontal line in (a) denote simulations in which a runaway greenhouse occurs.

\section{2) $\mathrm{FSC}$}

As we previously pointed out, the transition from the cold regime to the warm regime in FSC, which occurs as the TSI is increased to $1.25-1.30 S_{0}$, is made possible by the increasing albedo, which causes a decrease in absorbed solar radiation with increasing SST (as can also be seen in Fig. 7c). Since the OLR hardly increases with SST for SSTs above $340 \mathrm{~K}$, our results suggest that the decrease in absorbed solar radiation with increasing SST (caused by the clouds) is crucial for stable steady states to exist at all in the warm regime (Figs. 7c,d). Otherwise, the slope of the curve for the OLR could not be larger than the one for absorbed solar radiation. Furthermore, if the albedo saturated at a value below around 0.5 , for TSIs sufficiently large to destabilize the cold climate, the absorbed solar radiation would remain larger than the OLR for all SSTs we consider. Therefore, a transition from the cold regime to the warm regime would not be possible. In this case, the column would go directly from the cold regime into a runaway greenhouse that would, however, still occur at a larger TSI than for CSC. For the largest possible steady-state SST in the cold regime, the OLR is far away from its maximum, because the climate instability at this SST is caused by a positive shortwave cloud feedback, as evidenced by the large increase in absorbed solar radiation in Fig. 7c. This points to an important difference between CSC and FSC in how the cold regime is destabilized: In CSC, the decrease in OLR is the main cause of the climate instability, whereas in FSC, it is the cloud albedo effect. Hence, the character of climate instabilities changes fundamentally when clouds are included in the calculations.

\section{Sensitivity experiments}

In this section, we explore the sensitivity of our simulations to the choice of the parameterization of the scheme for fractional cloud cover, to the choice of molecular absorption coefficients in the longwave radiation scheme, and to the choice of the convective entrainment rate. This helps us to assess the robustness of important aspects of our results.

\section{a. Simulations with the Tompkins scheme for fractional cloud cover}

The Tompkins scheme for fractional cloud cover was already described in section 3a(4), and we will therefore directly discuss the main results. The most notable differences to the results obtained with the original scheme for fractional cloud cover (Sundqvist) are that the climate transition from the cold regime to the warm regime occurs at larger TSI, that the critical TSI to trigger a runaway greenhouse is larger, and that there is, in general, more cloud condensate in the warm regime in the simulations with the Tompkins scheme (Fig. 9). The qualitative behavior of these simulations is, however, similar to the one obtained with the original scheme for fractional cloud cover.

\section{$b$. Sensitivity to the entrainment rate of the convection scheme}

The entrainment rate of the convective updraft is one of the most effective tuning parameters in our singlecolumn model. An increase in entrainment rate leads to a larger influx of nonbuoyant air from the environment, in turn causing a faster decrease of buoyancy with altitude and therefore a shallower convective cell. In our 
(a)
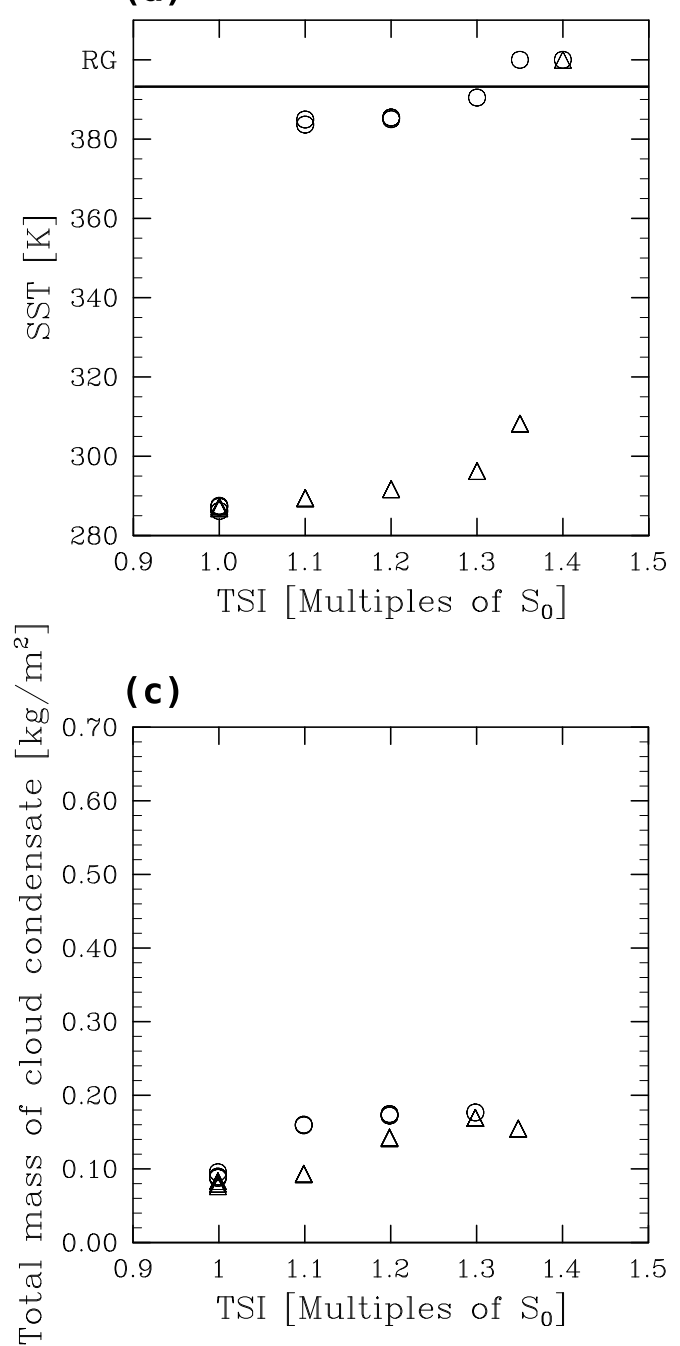

(b)

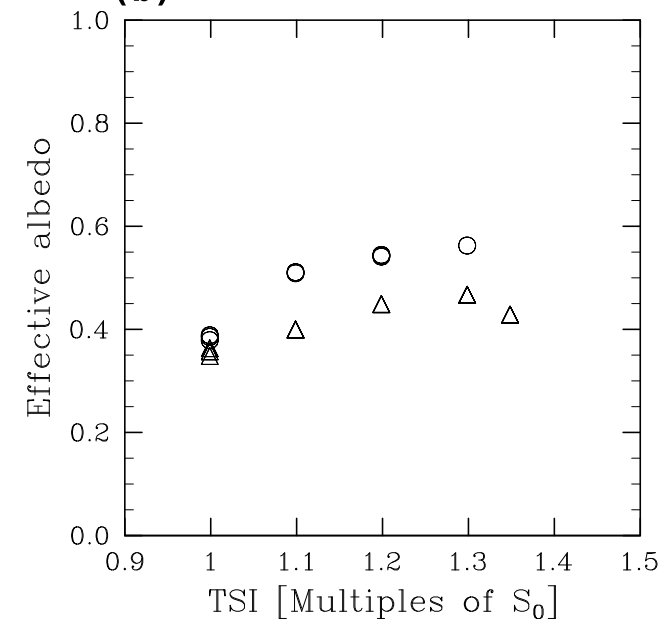

(d)

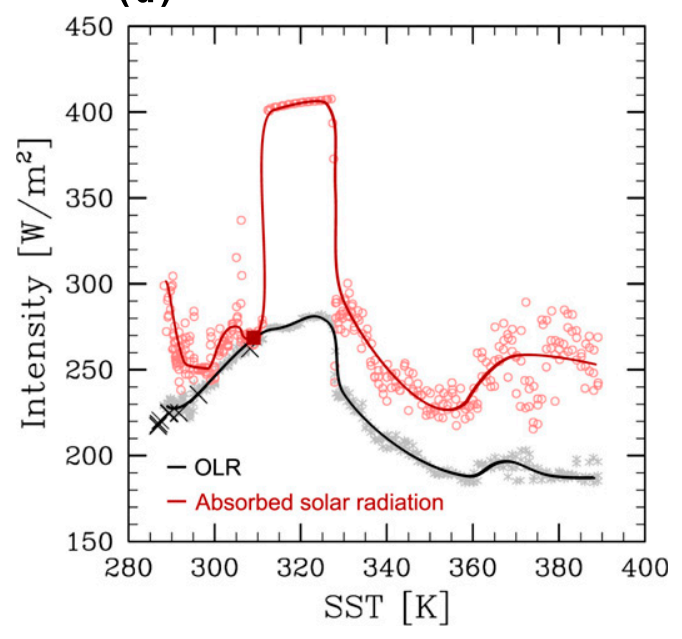

FIG. 10. Results from the simulations with the increased entrainment rate: Temporal mean over the last 5 years of simulation of (a) the SST, (b) the effective albedo, and (c) the vertically integrated cloud condensate with the increased entrainment rate as a function of TSI (the corresponding figures for the entrainment rate are Figs. 1b and 3). Triangles indicate simulations with a cold start, and circles indicate simulations with a warm start. Marks located above the horizontal line in (a) denote simulations in which a runaway greenhouse occurs. (d) A synthesis of OLR and of the net absorbed solar radiation as a function of SST for the simulations with a cold start. The large black crosses denote the OLR in steady states. Gray stars denote values of OLR taken from transient simulations at a spacing of $1 \mathrm{~K}$, and the black line is a manual fit to the gray stars. The red circles are obtained by multiplying values of the albedo from transient simulations with a fixed value of TSI of $1.30 S_{0}$ and, hence, approximate the total absorbed solar radiation for this TSI. The location of the steady state with this TSI is denoted by a red square. The red line is a manual fit to the red circles.

standard experiments, we choose an entrainment rate of $1 \times 10^{-4} \mathrm{~m}^{-1}$, which is a typical value for deep convection in ECHAM6 (Mauritsen et al. 2012). For the sensitivity experiments, we increase the entrainment rate to $3 \times 10^{-4} \mathrm{~m}^{-1}$, which is a typical value for shallow convection in ECHAM6 (Mauritsen et al. 2012). The qualitative behavior is again similar to the one obtained with the original entrainment rate in that there are double equilibria, the disappearance of low destabilizes the cold regime, cloud condensate and cloud albedo increase at SSTs above $340 \mathrm{~K}$, and the critical TSI is larger than for CSC. There is, however, one important difference: there is no transition from the cold regime to the warm regime, and the column goes directly from the cold regime into a runaway greenhouse (Fig. 10). There are two reasons why this does not occur. First, the 
(a)
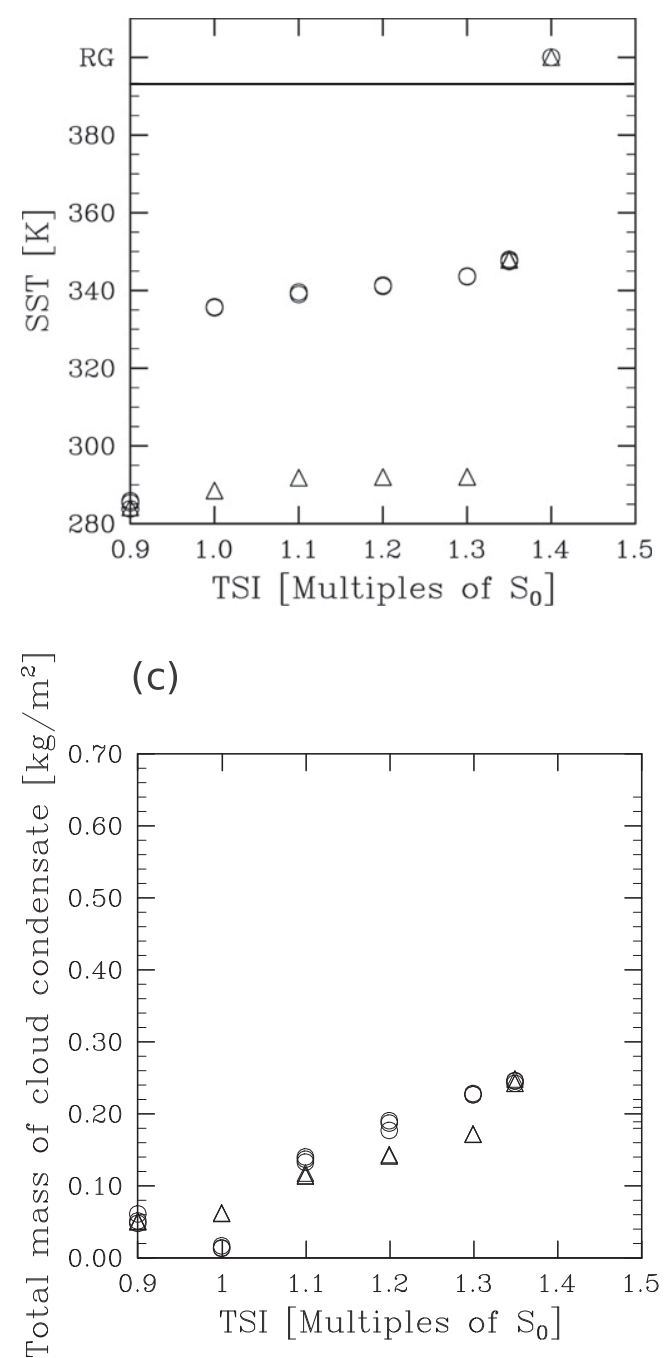

(b)

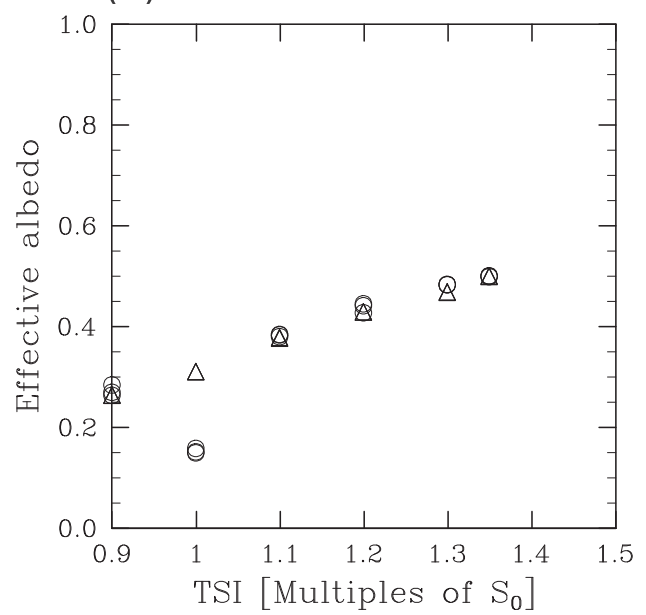

(d)

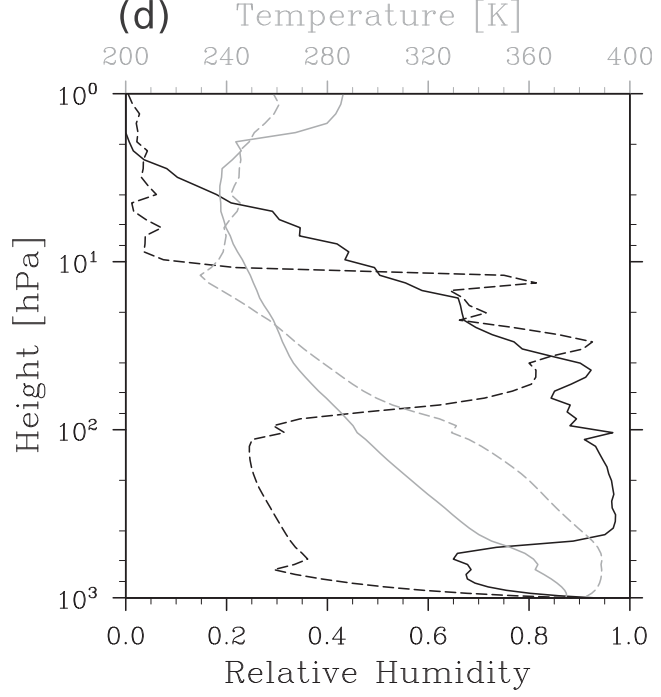

FIG. 11. Results from the simulations with the modified molecular absorption coefficients: Temporal mean over the last 5 years of simulation of (a) the SST, (b) the effective albedo, and (c) the vertically integrated cloud condensate with the Tompkins scheme as a function of TSI (the corresponding figures for the original set of molecular absorption coefficients are Figs. $1 \mathrm{~b}$ and 3). Triangles indicate simulations with a cold start, and circles indicate simulations with a warm start. Marks located above the horizontal line in (a) denote simulations in which a runaway greenhouse occurs. (d) The vertical profiles of temperature (gray lines) and relative humidity (black lines) before the breakdown of the radiative-convective equilibrium (solid lines) and after the breakdown (dashed lines). The height is given in terms of pressure of dry air.

critical TSI to destabilize the cold regime is larger than it is in the standard experiments; second, the critical TSI to destabilize the warm regime is smaller than in the standard experiments. In the previous section, we argued that the cloud albedo could saturate at a value that is too low for a steady state to be attained in the warm regime (at least for the range of SSTs that we consider in this study). This is exactly what happens in the experiments with the large entrainment rate: the absorbed solar radiation does not decrease sufficiently in the warm regime to allow for a steady state (Fig. 10d).

\section{c. Sensitivity to the molecular absorption coefficients for longwave radiation}

We perform a set of experiments with a modified extrapolation of the molecular absorption coefficients for the longwave radiation. These coefficients yield less absorption in the intermediate range of SST and a better fit to a state-of-the-art radiative transfer model than the original molecular absorption coefficients do (see the appendix). The results of these simulations are qualitatively similar to the standard experiments with FSC (Fig. 11). There is, 
however, one interesting difference compared to all other simulations. When the SST exceeds $375 \mathrm{~K}$, convection starts breaking down (not shown), the troposphere starts drying dramatically, and a large temperature inversion forms in the lower troposphere (Fig. 11d). This behavior is similar to the breakdown of radiativeconvective equilibrium for supercritical solar flux described by Rennó et al. (1994). The behavior is caused by the inability of the convection scheme to cool the surface sufficiently quickly. In this case, the runaway greenhouse occurs very quickly. Such a breakdown is not found in any of our other setups, and we doubt that a rapid breakdown is a physically plausible behavior, because all mass flux schemes have inherent limitations that put artificial upper bounds to the convective cooling of the surface.

\section{d. Summary}

The sensitivity experiments show that the quantitative results depend on the choice of the parameterizations, whereas the qualitative behavior is not affected strongly. Robust results are the existence of two equilibria, the disappearance of low clouds as the cause of destabilizing the cold regime, the increase of cloud condensate and cloud albedo at SSTs above $340 \mathrm{~K}$, and a critical TSI that is larger than for CSC. The results from the simulations with an increased convective entrainment rate suggest that, as the SSTs increase, the cloud albedo may not become large enough to allow simulations with a cold start to attain a steady state in the warm regime. However, even in this case, the critical TSI to cause a runaway greenhouse is still larger, even when starting in the warm regime, than the critical TSI required to cause a runaway greenhouse in CSC.

\section{Comparison to recent three-dimensional studies}

In the study by Boer et al. (2005), a climate instability occurred for a TSI of $1.35 S_{0}$. However, for this TSI, their model crashed at a mean SST increase of $50 \mathrm{~K}$. The absolute value of the mean SST hence did not exceed $340 \mathrm{~K}$. The climate instability leading to the rising SSTs in the study of Boer et al. (2005) was caused by a decrease in cloud albedo, an effect that also occurs in our study. However, it remains unclear whether the cloud albedo would recover in Boer et al. (2005), as seen in our study.

Several studies (e.g., Colman 2003; Bony et al. 2006; Soden et al. 2008) have analyzed and discussed the cloud feedbacks obtained by various GCM simulations with increased atmospheric $\mathrm{CO}_{2}$ concentrations. Depending on the GCM, the cloud feedback can be positive or negative, but it is positive in most cases. For increased solar forcing, Leconte et al. (2013) find a positive cloud feedback, whereas Wolf and Toon (2014) find a negative cloud feedback. In general, our model shows both behaviors with first a negative cloud feedback caused by an increase of both cover and amount of condensate of the boundary layer clouds and then a positive cloud feedback as the boundary layer clouds eventually disappear. Thus, the qualitative behaviors of Leconte et al. (2013) and Wolf and Toon (2014) do not necessarily need to be contradictory: it may simply be that the cloud feedback would become negative as well in Wolf and Toon (2014), but for larger values of TSI than in Leconte et al. (2013) (as seen in our model).

The results by Leconte et al. (2013) suggest that the atmosphere becomes increasingly dry with increasing equilibrium SST, especially in the subsidence region of the Hadley cell. Obviously our one-dimensional model is not able to capture such a dynamical effect. However, since the highest presented global-mean SST in that study is $340 \mathrm{~K}$, their results do not necessarily contradict ours, because the steady-state SSTs that we find in the warm regime are higher than $340 \mathrm{~K}$ when starting from a cold state. A moistening of their planet could simply occur at even higher temperatures. Another effect that may explain why the atmosphere in Leconte et al. (2013) is drier than in our model is the absence of land in our column. The closest three-dimensional analog to our column would be an aqua planet, rather than Earth, and for these two cases, clearly the aqua planet with the smaller global land fraction can be expected to be moister in the global mean than Earth.

\section{Conclusions}

Based on our simulations with a single-column version of the general circulation model ECHAM6, we conclude the following:

(i) Clouds provide a strong negative feedback and, hence, increase substantially the critical total solar irradiance that is necessary to trigger a runaway greenhouse. In our standard experiments, the critical value is increased from on the order of 1.15$1.20 S_{0}$ for clear-sky conditions (clouds are transparent to radiation) to $1.40-1.45 S_{0}$ for full-sky conditions (clouds are radiatively active).

(ii) When clouds are included in the radiative transfer calculations, climate instabilities are no longer caused by longwave effects but by the cloud albedo effect.

(iii) Clouds enlarge the range of total solar irradiance for which double equilibria are possible: in addition to states with near-present-day sea surface temperatures, 
(a)

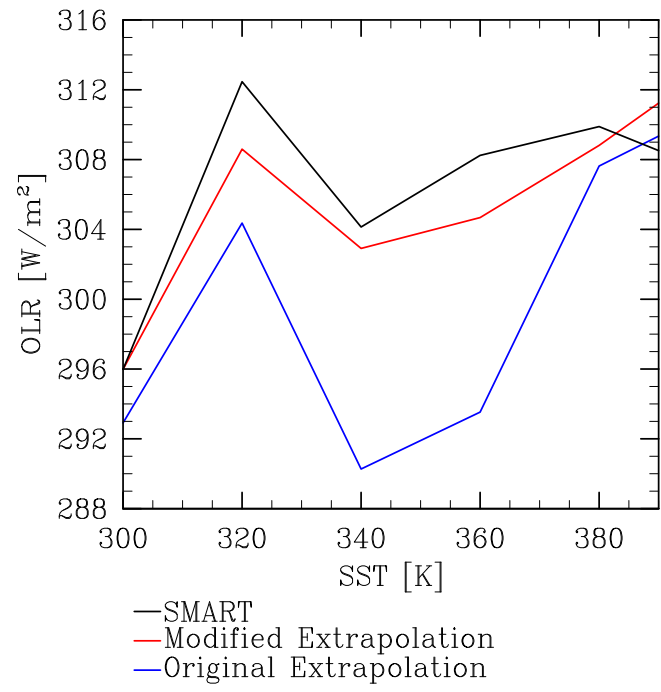

(c)

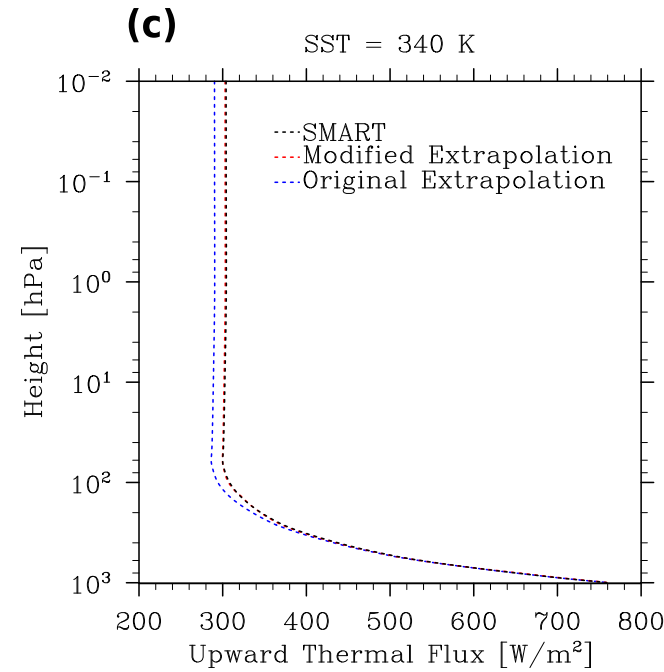

(b)

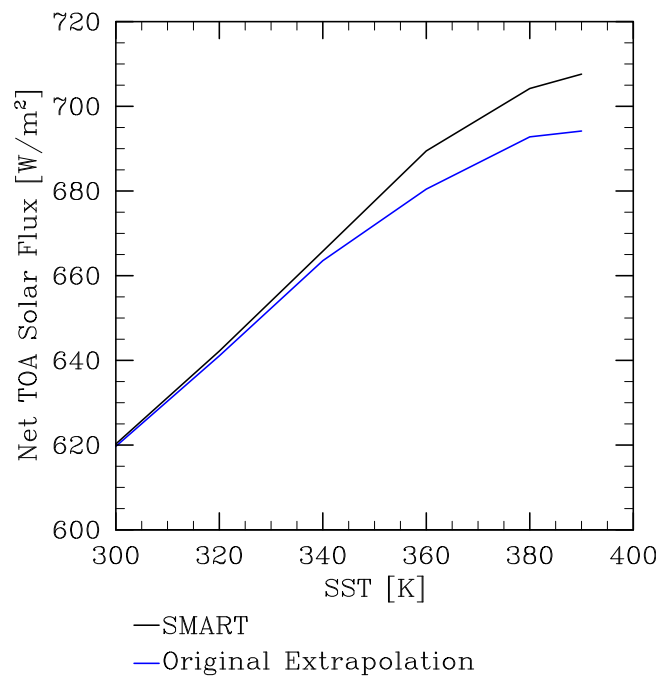

(d)

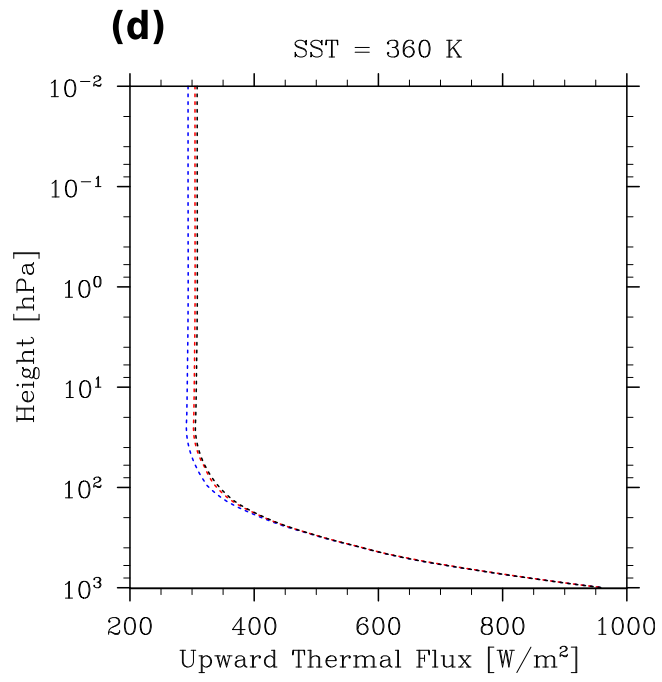

FIG. A1. Evaluation of the radiative transfer scheme. (a) A comparison of OLR as a function of SST between SMART (black), our radiative transfer scheme with the original extrapolation scheme (blue), and the modified extrapolation scheme (red). (b) The comparison of the net top-of-the-atmosphere solar flux as a function of SST for $1.0 S_{0}$ and a solar zenith angle of $52.5^{\circ}$ between SMART (black) and our radiative transfer scheme with the original extrapolation scheme (blue). Note that the atmospheric profiles are not taken from steady states. Also shown are the vertical profiles of the upward thermal flux for (c) SST $=340 \mathrm{~K}$ and (d) SST $=360 \mathrm{~K}$ for SMART (black), our radiative transfer scheme with the original extrapolation scheme (blue), and the modified extrapolation scheme (red). The vertical axis is the height in terms of pressure of dry air.

warm states exist with sea surface temperatures larger than $335 \mathrm{~K}$.

(iv) Thickening clouds do not reduce the outgoing longwave radiation anymore in the warm steady states, and therefore a further thickening of clouds increases only their shortwave effect. This mechanism allows the column to remain in balance at high sea surface temperatures and, furthermore, increases the critical total solar irradiance to trigger a runaway greenhouse.
Acknowledgments. We thank Erich Roeckner and Davide Zanchettin for valuable suggestions on earlier versions of the draft and Sebastian Rast and Suvarchal K. Cheedela for the technical support with the model. We express our gratitude to Colin Goldblatt, to an anonymous reviewer, and to the editor Ming Cai for their thorough reviews and for many valuable suggestions. Furthermore, we thank Colin Goldblatt for running SMART with our temperature profiles. We thank the International Max Planck Research School on Earth 
System Modelling and the Max Planck Society for the Advancement of Science for financial support.

\section{APPENDIX}

\section{Evaluation of the Radiative Transfer Code}

We have taken a few atmospheric profiles for states at different SSTs $(300,320,340,360,380$, and $390 \mathrm{~K})$ and compared the radiative fluxes obtained from our model to the ones obtained with the Spectral Mapping Atmospheric Transfer Model (SMART) (Meadows and Crisp 1996) in the setup described in Goldblatt et al. (2013) (courtesy of C. Goldblatt) (Fig. A1). The OLR is quite accurate for the lower and the upper range of SSTs, but the discrepancy grows to $15 \mathrm{~W} \mathrm{~m}^{-2}$ in the mid-300-K range. In general, our atmosphere absorbs too much of both longwave and shortwave radiation. Hence, the runaway greenhouse may occur at too-low TSI values for both CSC and FSC, and the warm regime of steady states may extend to even higher TSIs (at least for FSC). Water vapor absorption is poorly modeled in the upper levels of the original part of the model if water vapor becomes very abundant there. This is, however, of little significance for our study, because most of the upper atmosphere remains very dry.

To assess the influence of the too-strong infrared absorption in the mid-300-K temperatures, we have modified the radiative transfer code to yield a better match with SMART for the intermediate range of SST (but a slightly worse match at very high temperatures). For this version, we have linked the transition from tropospheric to stratospheric molecular absorption coefficients to the location of the tropopause (instead of having it fixed at $95 \mathrm{hPa}$ ), which yields much better results if water vapor becomes abundant in the upper atmosphere. Furthermore, we applied a heuristic blending function to the molecular absorption coefficients to obtain a better match to SMART (Fig. A1). We present the results obtained with the thus-modified molecular absorption coefficients in section $6 \mathrm{c}$.

\section{REFERENCES}

Abe, Y., A. Abe-Ouchi, N. H. Sleep, and K. J. Zahnle, 2011: Habitable zone limits for dry planets. Astrobiology, 11, 443460, doi:10.1089/ast.2010.0545.

Boer, G. J., K. Hamilton, and W. Zhu, 2005: Climate sensitivity and climate change under strong forcing. Climate Dyn., 24, 685700, doi:10.1007/s00382-004-0500-3.

Bony, S., and Coauthors, 2006: How well do we understand and evaluate climate change feedback processes? J. Climate, 19, 3445-3482, doi:10.1175/JCLI3819.1.

Brient, F., and S. Bony, 2013: Interpretation of the positive lowcloud feedback predicted by a climate model under global warming. Climate Dyn., 40, 2415-2431, doi:10.1007/ s00382-011-1279-7.

Clement, A. C., R. Burgman, and J. R. Norris, 2009: Observational and model evidence for positive low-level cloud feedback. Science, 325, 460-464, doi:10.1126/science.1171255.

Colman, R., 2003: A comparison of climate feedbacks in general circulation models. Climate Dyn., 20, 865-873, doi:10.1007/ s00382-003-0310-z.

Gold, T., 1964: Outgassing process on the Moon and Venus. The Origin and Evolution of Atmospheres and Oceans, P. Brancazio and A. G. W. Cameron, Eds., Wiley and Sons, 249-256.

Goldblatt, C., and A. J. Watson, 2012: The runaway greenhouse: Implications for future climate change, geoengineering and planetary atmospheres. Philos. Trans. Roy. Soc. London, A370, 4197-4216, doi:10.1098/rsta.2012.0004.

_ T. D. Robinson, K. J. Zahnle, and D. Crisp, 2013: Low simulated radiation limit for runaway greenhouse climates. Nat. Geosci., 6, 661-667, doi:10.1038/ngeo1892.

Heinemann, M., 2009: Warm and sensitive Paleocene-Eocene climate. Max Planck Institute Tech. Rep. 70/2009, 119 pp. [Available online at http://www.mpimet.mpg.de/fileadmin/ publikationen/Reports/WEB_BzE_70.pdf.]

Iacono, M. J., J. S. Delamere, E. J. Mlawer, M. W. Shephard, S. A. Clough, and W. D. Collins, 2008: Radiative forcing by longlived greenhouse gases: Calculations with the AER radiative transfer models. J. Geophys. Res., 113, D13103, doi:10.1029/ 2008JD009944.

Ingersoll, A. P., 1969: The runaway greenhouse: A history of water on Venus. J. Atmos. Sci., 26, 1191-1198, doi:10.1175/ 1520-0469(1969)026<1191:TRGAHO > 2.0.CO;2.

Kasting, J. F., 1988: Runaway and moist greenhouse atmospheres and the evolution of Earth and Venus. Icarus, 74, 472-494, doi:10.1016/0019-1035(88)90116-9.

— , R. Kopparapu, R. M. Ramirez, and C. E. Harman, 2014: Remote life-detection criteria, habitable zone boundaries, and the frequency of Earth-like planets around $\mathrm{M}$ and late K stars. Proc. Natl. Acad. Sci. USA, 111, 12 641-12646, doi:10.1073/ pnas.1309107110.

Komabyashi, M., 1967: Discrete equilibrium temperatures of a hypothetical planet with the atmosphere and the hydrosphere of one component-two phase system under constant solar radiation. J. Meteor. Soc. Japan, 45, 137-139.

Kopparapu, R. K., and Coauthors, 2013: Habitable zones around main-sequence stars: New estimates. Astrophys. J., 765, 131, doi:10.1088/0004-637X/765/2/131.

Leconte, J., F. Forget, B. Charnay, R. Wordsworth, and A. Pottier, 2013: Increased insolation threshold for runaway greenhouse processes on Earth-like planets. Nature, 504, 268-271, doi:10.1038/nature12827.

Lohmann, U., and E. Roeckner, 1996: Design and performance of a new cloud microphysics scheme developed for the ECHAM general circulation model. Climate Dyn., 12, 557-572, doi:10.1007/BF00207939.

Mauritsen, T., and Coauthors, 2012: Tuning the climate of a global model. J. Adv. Model. Earth Syst., 4, M00A01, doi:10.1029/ 2012MS000154.

Meadows, V. S., and D. Crisp, 1996: Ground-based near-infrared observations of the Venus nightside: The thermal structure and water abundance near the surface. J. Geophys. Res., 101, 4595-4622, doi:10.1029/95JE03567.

Mlawer, E. J., S. J. Taubman, P. D. Brown, M. J. Iacono, and S. A. Clough, 1997: Radiative transfer for inhomogeneous atmospheres: RRTM, a validated correlated-k model for the 
longwave. J. Geophys. Res., 102, 16663-16682, doi:10.1029/ 97JD00237.

Nakajima, S., Y.-Y. Hayashi, and Y. Abe, 1992: A study on the "runaway greenhouse effect" with a one-dimensional radiativeconvective equilibrium model. J. Atmos. Sci., 49, 2256-2266, doi:10.1175/1520-0469(1992)049<2256:ASOTGE>2.0.CO;2.

Nordeng, T. E., 1994: Extended versions of the convective parametrization scheme at ECMWF and their impact on the mean and transient activity of the model in the tropics. ECMWF Tech. Memo. 206, 41 pp.

Pierrehumbert, R. T., 2010: Principles of Planetary Climate. Cambridge University Press, $680 \mathrm{pp}$.

Pujol, T., and G. R. North, 2002: Runaway greenhouse effect in a semigray radiative-convective model. J. Atmos. Sci., 59, 28012810, doi:10.1175/1520-0469(2002)059<2801:RGEIAS $>2.0$. CO;2.

Rennó, N. O., 1997: Multiple equilibria in radiativeconvective atmospheres. Tellus, 49A, 423-438, doi:10.1034/ j.1600-0870.1997.t01-3-00002.x.

- P. H. Stone, and K. A. Emanuel, 1994: Radiative-convective model with an explicit hydrologic cycle: 2 . Sensitivity to large changes in solar forcing. J. Geophys. Res., 99, 17001-17020, doi:10.1029/94JD01332.

Roeckner, E., and Coauthors, 2003: The atmospheric general circulation model ECHAM5. Part I: Model description. MPI Tech. Rep. 349, 140 pp. [Available online at http://www. mpimet.mpg.de/en/wissenschaft/modelle/echam.html.]
Soden, B. J., I. M. Held, R. Colman, K. M. Shell, J. T. Kiehl, and C. A. Shields, 2008: Quantifying climate feedbacks using radiative kernels. J. Climate, 21, 3504-3520, doi:10.1175/ 2007JCLI2110.1.

Stevens, B., and Coauthors, 2013: Atmospheric component of the MPI-M Earth System Model: ECHAM6. J. Adv. Model. Earth Syst., 5, 146-172, doi:10.1002/jame.20015.

Sundqvist, H., E. Berge, and J. E. Kristjánsson, 1989: Condensation and cloud parameterization studies with a mesoscale numerical weather prediction model. Mon. Wea. Rev., 117, 1641-1657, doi:10.1175/1520-0493(1989)117<1641:CACPSW>2.0.CO;2.

Tiedtke, M., 1989: A comprehensive mass flux scheme for cumulus parameterization in large-scale models. Mon. Wea. Rev., 117, 17791800, doi:10.1175/1520-0493(1989)117<1779:ACMFSF $>2.0 . C O ; 2$.

Tompkins, A. M., 2002: A prognostic parameterization for the subgrid-scale variability of water vapor and clouds in largescale models and its use to diagnose cloud cover. J. Atmos. Sci., 59, 1917-1942, doi:10.1175/1520-0469(2002)059<1917: APPFTS $>2.0 . \mathrm{CO} ; 2$.

Vardavas, I. M., and J. H. Carver, 1985: Atmospheric temperature response to variations in $\mathrm{CO}_{2}$ concentration and the solarconstant. Planet. Space Sci., 33, 1187-1207, doi:10.1016/ 0032-0633(85)90076-5.

Wolf, E. T., and O. B. Toon, 2014: Delayed onset of runaway and moist greenhouse climates for Earth. Geophys. Res. Lett., 41, 167-172, doi:10.1002/2013GL058376. 